\title{
Search for the Higgs boson in the all-hadronic final state using the full CDF data set
}

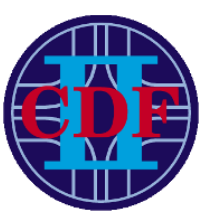

\section{The CDF collaboration}

E-mail: mitra@fnal.gov

ABSTRACT: This paper reports the result of a search for the standard model Higgs boson in events containing four reconstructed jets associated with quarks. For masses below $135 \mathrm{GeV} / \mathrm{c}^{2}$, the Higgs boson decays to bottom-antibottom quark pairs are dominant and result primarily in two hadronic jets. An additional two jets can be produced in the hadronic decay of a $W$ or $Z$ boson produced in association with the Higgs boson, or from the incoming quarks that produced the Higgs boson through the vector-boson fusion process. The search is performed using a sample of $\sqrt{s}=1.96 \mathrm{TeV}$ proton-antiproton collisions corresponding to an integrated luminosity of $9.45 \mathrm{fb}^{-1}$ recorded by the CDF II detector. The data are in agreement with the background model and $95 \%$ credibility level upper limits on Higgs boson production are set as a function of the Higgs boson mass. The median expected (observed) limit for a $125 \mathrm{GeV} / c^{2}$ Higgs boson is 11.0 (9.0) times the predicted standard model rate.

KEYworDs: Hadron-Hadron Scattering

ARXiv EPRINT: 1208.6445 


\section{Contents}

1 Introduction 1

2 The CDF II detector 2

3 Event selection 3

$4 \quad$ Signal and background samples 4

5 Search strategy 5

$\begin{array}{lll}6 & \text { QCD multi-jet background prediction } & 6\end{array}$

$\begin{array}{lll}7 & \text { Energy correction for } b \text { jets } & 7\end{array}$

8 Untagged jets neural network $\quad 8$

9 Jet width 9

10 Classification of Higgs boson events 10

11 Systematic uncertainties $\quad 16$

12 Results $\quad 18$

$\begin{array}{ll}13 \text { Summary } & 19\end{array}$

$\begin{array}{ll}\text { The CDF collaboration } & 23\end{array}$

\section{Introduction}

The Higgs boson is the physical manifestation of the hypothesized mechanism that provides mass to fundamental particles in the standard model (SM) [1-3]. Direct searches at the LEP collider [4], the Tevatron [5], and the LHC [6, 7] have excluded SM Higgs boson masses at the $95 \%$ confidence level or $95 \%$ credibility level (CL), except within the range $122-128 \mathrm{GeV} / c^{2}$. The most sensitive searches at the LHC are based on SM Higgs boson decays to pairs of gauge bosons. At the Tevatron, searches based on Higgs boson decay to bottom-antibottom quark pairs $(b \bar{b})$ are the most sensitive within the allowed range. Searches in this channel offers complementary information on fermion Yukawa couplings to the Higgs boson. 
Recently, the ATLAS and CMS collaborations have reported the observation of a Higgslike particle at a mass of $\approx 125 \mathrm{GeV} / c^{2}[6,7]$, and the Tevatron has reported evidence for a particle decaying to $b \bar{b}$ produced in association with a $W / Z$ boson for masses within the range $120-135 \mathrm{GeV} / c^{2}[8]$.

This paper describes a search for the Higgs boson using a data sample corresponding to an integrated luminosity of $9.45 \mathrm{fb}^{-1}$ of $p \bar{p}$ collisions at $\sqrt{s}=1.96 \mathrm{TeV}$ recorded by the Collider Detector at Fermilab (CDF II). In this search two production mechanisms are studied: associated vector-boson production $(V H)$ and vector-boson fusion (VBF). The $V H$ channel denotes the process $p \bar{p} \rightarrow W / Z+H \rightarrow q \bar{q}^{\prime}+b \bar{b}$. The VBF channel identifies the process $p \bar{p} \rightarrow q \bar{q}^{\prime} H \rightarrow q \bar{q}^{\prime} b \bar{b}$, where the two incoming quarks each radiate a weak boson, which subsequently fuse into a Higgs boson. In both channels, the Higgs boson decays to $b \bar{b}$, and is produced in association with two other quarks $\left(q \bar{q}^{\prime}\right)$. Data are tested against the hypothesis of the presence of Higgs boson with mass in the range $100<m_{H}<150 \mathrm{GeV} / c^{2}$. The $H \rightarrow b \bar{b}$ mode is the dominant decay for $m_{H}<135 \mathrm{GeV} / c^{2}[9]$.

Searches for a Higgs boson performed in final states containing leptons, jets, and missing energy have the advantage of smaller background, but the Higgs boson signal yields are also very small. The all-hadronic search channel, described here, has larger potential signal contributions but suffers from substantial QCD multi-jet background contributions. The challenge of this channel is to accurately model and reduce the multi-jet background. Two previous papers were published on searches for a Higgs boson in the all-hadronic channel at CDF using data sets of $2 \mathrm{fb}^{-1}$ [10] and $4 \mathrm{fb}^{-1}$ [11]. Another paper was published on searches for a Higgs boson in the all-hadronic channel at CDF using data collected during Run I [12]. The LEP collider also conducted searches for the Higgs boson in the all-hadronic final state in the $e^{+} e^{-} \rightarrow Z H \rightarrow q \bar{q}+b \bar{b}$ channel [4].

\section{The CDF II detector}

The CDF II detector is an azimuthally and forward-backward symmetric multipurpose detector. CDF II uses a cylindrical coordinate system with the $z$-axis aligned along the proton beam direction, where $\theta$ is the polar angle relative to the $z$-axis and $\phi$ is the azimuthal angle relative to the $x$-axis. The pseudorapidity is defined as $\eta=-\ln (\tan \theta / 2)$ and the transverse energy is calculated as $E_{T}=E \sin \theta$.

The CDF II detector consists of a pair of concentric charged-particle tracking detectors immersed in a $1.4 \mathrm{~T}$ solenoid magnetic field, surrounded by calorimeters and muon detectors. The inner tracking detector is the silicon vertex detector that is located immediately outside the beam pipe, provides precise three-dimensional reconstruction of charged-particle trajectories (tracks) and is used to identify displaced vertices associated with bottom-quark and charm-quark hadron decays. The momenta of charged particles is measured precisely in the central outer tracker (COT), a cylindrical multiwire drift chamber. The tracking detectors cover the pseudorapidity range $|\eta|<1.1$. Outside the COT are electromagnetic and hadronic calorimeters arranged in a projective-tower geometry, covering the region $|\eta|<3.5$, to provide energy measurements for both charged and neutral particles. Drift chambers and scintillator counters in the region $|\eta|<1.5$ provide muon 
identification outside the calorimeters. Luminosity is measured using low-mass gaseous Cherenkov luminosity counters (CLC). There are two CLC modules in the CDF II detector installed at small angles in the proton and antiproton directions, arranged in three concentric layers around the beam pipe. More details about the CDF II detector can be found in refs. [13-15].

Jets are defined by a cluster of energy deposited in the calorimeter using a jet clustering algorithm (JetClu) [16] with a cone of fixed radius. The JetClu algorithm begins by creating a list of calorimeter towers above a fixed $E_{T}$ threshold to be used as seeds for the jet finder. This threshold is set to $1.0 \mathrm{GeV}$. Preclusters are formed from an unbroken chain of contiguous seed towers with a continuously decreasing tower $E_{T}$. If a tower is outside a window of seven towers surrounding the seed, it is used to form a new precluster. These preclusters are used as a starting point for cone clustering. First, the $E_{T}$ weighted centroid of the precluster is found and a cone in $\eta-\phi$ space of radius $R$ is formed around the centroid. For this analysis, $\Delta R=\sqrt{(\Delta \phi)^{2}+(\Delta \eta)^{2}}=0.4$. Then, all towers with an $E_{T}$ of, at least, $100 \mathrm{MeV}$ are incorporated into the cluster. A tower is included in a cluster if its centroid is inside the cone, otherwise it is excluded. A new cluster center is calculated from the set of towers within the clustering cone, again using an $E_{T}$ weighted centroid, and a new cone is drawn about this position. The process of recomputing a centroid and finding new or deleting old towers is iterated until the tower list remains unchanged. Corrections are applied to the measured jet energy to account for detector calibrations, multiple interactions, underlying event, and energy outside of the jet cone [17].

The data for this analysis are collected using two online event selections (triggers). Events in the first $3.0 \mathrm{fb}^{-1}$ are triggered by selecting those containing at least four jets with $E_{T} \geq 15 \mathrm{GeV}$ and total calorimeter $E_{T}$ greater than $175 \mathrm{GeV}$. Events in the remaining 6.45 $\mathrm{fb}^{-1}$ are selected by requiring at least three jets with $E_{T} \geq 20 \mathrm{GeV}$ and total calorimeter $E_{T}$ greater than $130 \mathrm{GeV}$.

\section{Event selection}

Events with isolated leptons or missing transverse energy significance ${ }^{1}$ greater than 6.0 , which is indicative of the presence of neutrinos, are removed to ensure an event sample independent from other Higgs boson searches at CDF. Events containing four or five jets, with $E_{T}>15 \mathrm{GeV}$ and $|\eta|<2.4$ are selected.

To reduce the QCD multi-jet background, exactly two bottom-quark jets ( $b$ jets) are required. Two algorithms are used to identify $b$ jets: the SecVtx algorithm [14] and the JetProb algorithm [18]. The SecVtx algorithm attempts to reconstruct the secondary vertex associated with a bottom-quark (b) decay. The JetProb algorithm searches the impact parameter of the charged-particle trajectories (tracks) within a jet and selects those that are inconsistent with originating from the decay of a particle occurred in the vicinity

\footnotetext{
${ }^{1}$ Missing transverse energy significance is defined as the ratio of the missing transverse energy to the square root of the total transverse energy. The missing transverse energy, $E_{T}=\left|\vec{E}_{T}\right|$, where $\vec{E}_{T}$ is defined by, $\vec{E}_{T}=-\sum_{i} E_{T}^{i} \hat{n}_{i}$, where $i$ is calorimeter tower number with $|\eta|<3.6, \hat{n}_{i}$ is a unit vector perpendicular to the beam axis and pointing at the $\mathrm{i}^{\text {th }}$ calorimeter tower.
} 
of the primary event vertex. An additional energy correction is applied to jets identified as $b$ jets (section 7 ). Untagged jets (non $b$ jets) are referred to as $q$ jets in this paper.

All selected jets are ordered in $E_{T}$ and the four highest $E_{T}$ jets are considered. The scalar sum of the four selected jet $E_{T} \mathrm{~s}$ (SumEt) is required to exceed $220 \mathrm{GeV}$ and two of the four selected jets must be $b$ jets.

The signal-to-background ratio is enhanced by dividing the data into two independent $b$-tagging categories: SS in which both jets are tagged by SecVtx, and SJ in which one jet is tagged by SecVtx and the other by JetProb. If a jet is tagged by both algorithms, it is classified as tagged by SecVtx because of the lower misidentification rate. Events in which both jets are tagged only by JetProb are not used because the increase in background contributions is substantially larger than that for the signal.

The signal region is defined by requirements on the invariant mass of the two $b$-tagged jets $\left(m_{b b}\right)$ and the two untagged jets $\left(m_{q q}\right)$. The $V H$ channel features two intermediate resonances, one from the potential Higgs boson decay, in $m_{b b}$, and another from the $W / Z$ decay, in $m_{q q}$. The VBF channel shares the same $m_{b b}$ resonance but the two $q$ jets are not produced from the decay of a particle. However these two $q$ jets tend to be produced with large $\eta$ separation which gives a large effective $m_{q q}$ mass. The Higgs boson search region is defined as $75<m_{b b}<175 \mathrm{GeV} / c^{2}$ and $m_{q q}>50 \mathrm{GeV} / c^{2}$.

\section{Signal and background samples}

Backgrounds that contribute to the $q q b \bar{b}$ final state originate from QCD multi-jet production, top-quark pair production, single-top-quark production, $W \rightarrow q^{\prime} \bar{q}$ plus $b \bar{b}$ or charm-quark pair $(c \bar{c})$ production $(W+H F), Z \rightarrow b \bar{b}, c \bar{c}$ plus jets production ( $Z+$ jets), and diboson production $(W W, W Z, Z Z)$. About $98 \%$ of the total background comes from QCD multi-jet production. Signal and non-QCD backgrounds yields are estimated from Monte Carlo (MC) simulation. The $W+H F$ and $Z+$ jets contributions are modeled with the ALPGEN [19] generator for simulating the bosons plus parton production, and PYTHIA [20] for modeling parton showers. The other non-QCD backgrounds and the signal are modeled with PYTHIA [20]. All MC-simulated samples use the CTEQ5L [21] parton distribution function (PDF) at leading order (LO) and are processed through the full CDF II detector simulation [22], based on GEANT [23], that includes the trigger simulation and their trigger efficiencies are corrected as described in ref. [10].

The expected signal yield in the SS (SJ) channel is $27.1 \pm 4.1(9.1 \pm 1.4)$ for $m_{H}=$ $125 \mathrm{GeV} / c^{2}$. The selected number of data events for SS (SJ) are 87272 (46818). A datadriven model is used to predict the shape of QCD multi-jet background but not the overall yield (section 6). The number of QCD multi-jet events in each channel is estimated as the difference between the number of data events and the predicted number of non-QCD events estimated with MC (neglecting the potential Higgs boson contribution). Expected and observed event yields are summarized in table 1. In the final fit used to extract a potential Higgs boson signal, the overall normalization of the QCD multi-jet background is treated as an unconstrained parameter. 


\begin{tabular}{|l|c|c|}
\hline Backgrounds & SS channel & SJ channel \\
\hline$t \bar{t}$ & $1032 \pm 156$ & $384 \pm 57$ \\
Single top $s$ channel & $111 \pm 19$ & $38 \pm 6$ \\
Single top $t$ channel & $44 \pm 7$ & $26 \pm 4$ \\
$W+b \bar{b}$ & $77 \pm 40$ & $29 \pm 15$ \\
$W+c \bar{c}$ & $8 \pm 4$ & $7 \pm 4$ \\
$Z(\rightarrow b \bar{b} / c \bar{c})+$ jets & $873 \pm 452$ & $338 \pm 175$ \\
$W W$ & $6 \pm 1$ & $6 \pm 1$ \\
$W Z$ & $20 \pm 3$ & $8 \pm 1$ \\
$Z Z$ & $21 \pm 3$ & $8 \pm 1$ \\
\hline Total non-QCD & $2192 \pm 480$ & $844 \pm 185$ \\
\hline \hline Data & 87272 & 46818 \\
\hline QCD multi-jet & 85080 & 45974 \\
\hline \hline Higgs signal $\left(125 \mathrm{GeV} / c^{2}\right)$ & $27 \pm 4$ & $9 \pm 1$ \\
\hline
\end{tabular}

Table 1. Expected number of background and signal $\left(m_{H}=125 \mathrm{GeV} / c^{2}\right)$ events that pass the complete event selection for the SS and SJ $b$-tag categories. The number of QCD multi-jet events is estimated as the difference between data and predicted non-QCD backgrounds (neglecting the potential Higgs contribution). The uncertainties of the signal and non-QCD background rate predictions include statistical and systematic rate uncertainties, such as cross-section and integrated luminosity, as described in section 11.

\section{$5 \quad$ Search strategy}

The main challenge is to accurately model and reduce the QCD multi-jet background. The modeling of this background is obtained from a data-driven technique described in section 6. This avoids the need of generating large volumes of QCD multi-jet simulation samples, which is computationally intensive and unlikely to accurately reproduce the multijet spectrum.

The overwhelming QCD multi-jet background is suppressed by relying on multi-variate techniques that combine information from multiple variables to identify potential Higgs boson events. For example, the best signal-to-background ratio using just $m_{b b}$ is $0.0007 .{ }^{2}$ In this search, the use of multi-variate techniques improves the signal-to-background to $0.006,{ }^{3}$ a factor of 10 improvement. A total of eleven artificial neural networks (NN) [24, 25] are used to improve the resolutions of variables sensitive to Higgs production and to separate the signal and background contributions. Altogether, the use of these NN leads to a $24 \%$ increase in search sensitivity, ${ }^{4}$ in addition to that expected from the inclusion of additional data with respect to the previous analysis [11].

This analysis focuses on Higgs boson decays to $b \bar{b}$, and thus it is important to have the best possible resolution for $m_{b b}$. Section 7 describes a NN used to correct the energies of

\footnotetext{
${ }^{2}$ Only considering events under the Higgs signal peak $\left(120<m_{b b}<140 \mathrm{GeV} / c^{2}\right)$, figure 3 (a).

${ }^{3}$ Only considering events with Higgs-NN $>0.95$, figure 8 .

${ }^{4}$ The search sensitivity is defined as the percentage reduction of the median expected limit.
} 
$b$ jets, which in turn improves $m_{b b}$. The untagged jets ( $q$ jets) associated with each Higgs production process have unique angular and kinematic distributions. Section 8 describes three networks that exploit these variables to identify $q$ jets from Higgs boson events. As gluon jets are typically wider than quark jets, jet width is useful for separating events containing quark jets associated with Higgs-boson production from generic jets contained within QCD multi-jet events, which are a mixture of quark and gluon jets. Section 9 describes a technique for measuring jet width and a NN used to remove detector and kinematic dependences that also influence the jet width.

Section 10 describes the final two-stage NN that is used to extract a potential signal contribution from the backgrounds. The two-stage NN can identify Higgs bosons produced by three different processes simultaneously. The first stage is based on three separate NNs trained specifically to separate backgrounds from either $W H, Z H$, or VBF Higgs production, respectively, to exploit the unique characteristics of each signal process. The outputs of the three process-specific NNs are used as inputs to a second NN. The inputs to the firststage networks are the corrected $b$-jet energies, corrected $q$-jet widths, outputs of the $q$-jet networks, and other kinematic event variables. In the previous search [11], exclusive $V H$ and VBF networks were used to search for Higgs bosons in non-overlapping signal regions. The two-stage NN, developed for this search, increases the search sensitivity by $15 \%$. The use of a single signal region increases the number of potential Higgs boson signal events by $20 \%$. Both gains are above those expected from the inclusion of additional data alone.

\section{QCD multi-jet background prediction}

Kinematic features of the QCD multi-jet background are predicted using a data-driven method. An independent data control region is used to measure the probability for an event with one $b$-tagged jet to contain an additional $b$-tagged jet (probe jet), referred to as the Tag Rate Function (TRF). The TRF is applied to data samples with exactly one jet $b$-tagged by SecVtx to predict the distribution of events with two $b$-tagged jets. The TRF is parameterized as a function of three variables: $E_{T}$ of the probe jet, $\eta$ of the probe jet, and $\Delta R$ between the tagged $b$ jet and probe jet, and implemented as a threedimensional histogram. The choice of variables used to parameterize the TRF is motivated by the kinematics of the QCD multi-jet background and the characteristics of the $b$-tagging algorithms. For example, the production of $b$ jets from gluon splitting has a different $\Delta R$ distribution compared to direct production, and the probe jet $E_{T}$ and $\eta$ expresses aspects of the $b$-tagging algorithms and QCD multi-jet production. Further information on the technique can be found in [10].

We use separate TRFs for SS and SJ events, which are obtained from events in the TAG region (figure 1), defined as $m_{q q} \in\left[40 \mathrm{GeV} / c^{2}, 45 \mathrm{GeV} / c^{2}\right] \cup m_{b b} \in\left[65 \mathrm{GeV} / c^{2}, 250 \mathrm{GeV} / c^{2}\right]$ and $m_{q q}>45 \mathrm{GeV} / c^{2} \cup m_{b b} \in\left[65 \mathrm{GeV} / c^{2}, 70 \mathrm{GeV} / c^{2}\right] \cup m_{b b} \in\left[200 \mathrm{GeV} / c^{2}, 250 \mathrm{GeV} / c^{2}\right]$. To validate the background model, the TRF is tested in the TAG (for self-consistency) and two other control regions non-overlapping with the signal region (figure 1): the CONTROL region, defined as $m_{q q} \in\left[45 \mathrm{GeV} / c^{2}, 50 \mathrm{GeV} / c^{2}\right] \cup m_{b b} \in\left[70 \mathrm{GeV} / c^{2}, 200 \mathrm{GeV} / c^{2}\right]$ and $m_{q q}>50 \mathrm{GeV} / c^{2} \cup m_{b b} \in\left[70 \mathrm{GeV} / c^{2}, 75 \mathrm{GeV} / c^{2}\right] \cup m_{b b} \in\left[175 \mathrm{GeV} / c^{2}, 200 \mathrm{GeV} / c^{2}\right] ;$ 


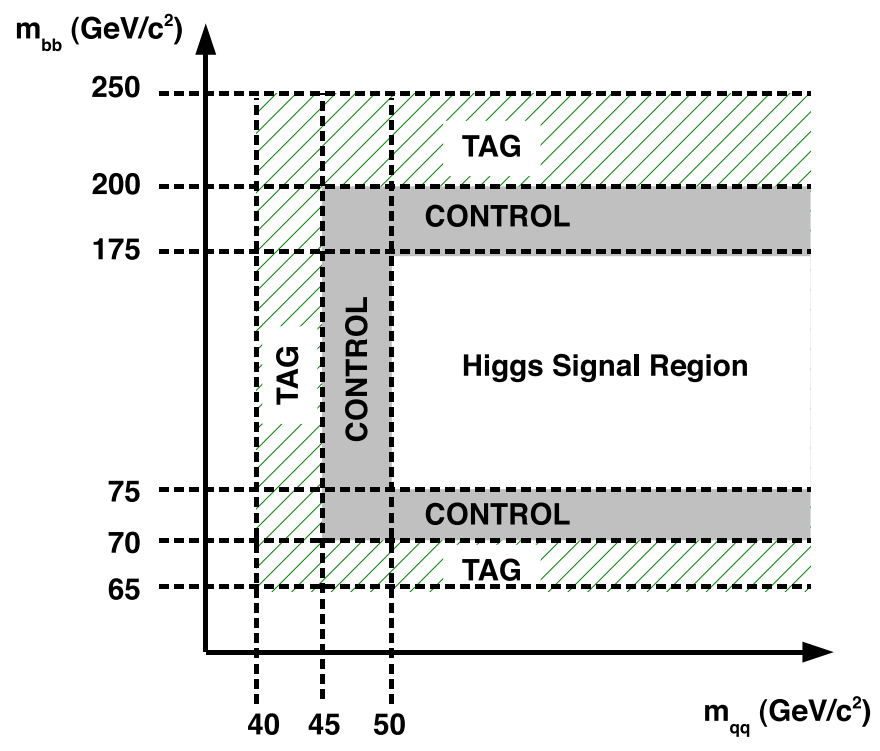

Figure 1. Signal and controls regions in the $m_{b b}-m_{q q}$ plane. The TAG region is used to derive the TRF for modeling the QCD multi-jet background. The CONTROL region is used to test and derive systematic uncertainties of this background model.

and the NJET6 control region defined as sharing the same $m_{b b}$ and $m_{q q}$ criteria as the signal region, but contains those events with six reconstructed jets. The TRF prediction of different variables are compared to data in these control regions and any shape difference is propagated as an uncertainty of the QCD multi-jet model.

The $m_{q q}$ variable is not perfectly modeled by the TRF. The residual mismodeling is corrected by following the procedure defined in previous searches $[10,11]$, which reweights events as a function of the observed $m_{q q}$. The correction function is derived from a fit to the ratio of the observed $m_{q q}$ over the same quantity predicted by TRF in events from the TAG region.

Figures 3-6 show a comparison of observed data and background predictions in the signal region for the variables used in the final signal discrimination neural network (section 10) after application of the $m_{q q}$ correction function. The modeling of some variables appears to be poor but the differences are within the shape uncertainties of the QCD multi-jet prediction. More details on these variables are given in the following sections.

\section{$7 \quad$ Energy correction for $b$ jets}

The experimental resolution of the invariant mass of the two $b$ jets, $m_{b b}$, has a significant effect on the sensitivity of our search. To improve the $m_{b b}$ resolution, a NN is trained to estimate the correction factor required to obtain the best possible estimate of the parent $b$-parton energy from the measured jet energy [26].

A NN is trained for each $b$-tagging algorithm. Nine variables, describing a given jet, are used to train the NN for SecVtx tagged jets. These are the jet $E_{T}$, the jet transverse 
momentum $\left(p_{T} \equiv p \sin \theta\right)$, the $E_{T}$ before the application of jet energy correction (uncorrected jet $E_{T}$ ), the transverse mass, ${ }^{5}$ the decay length of the jet in the transverse plane ${ }^{6}$ and its uncertainty, the $p_{T}$ of the secondary vertex, the maximum $p_{T}$ of the tracks inside the jet cone, and the $p_{T}$ sum of all tracks within the jet cone. Six variables are used to train the NN for JetProb tagged jets: the jet $E_{T}$, the jet $p_{T}$, the uncorrected jet $E_{T}$, the transverse mass, the maximum $p_{T}$ of the tracks inside the jet cone, and the $p_{T}$ sum of all tracks within the jet cone.

The NNs are trained using simulated VBF events ${ }^{7}$ with Higgs masses from $100 \mathrm{GeV} / c^{2}$ to $150 \mathrm{GeV} / c^{2}$ at $5 \mathrm{GeV} / c^{2}$ intervals. Events are required to pass the selection described in section 3 and each $b$-tagged jet is required to be matched geometrically with a $b$ parton. The matching criterion requires the $\Delta R$ between the $b$ jet and $b$ parton not to exceed 0.4. SecVtx- and JetProb-tagged jets are used to train the SecVtx and JetProb networks, respectively.

Figure 2 shows the $m_{b b}$ distribution in simulated decays of $125 \mathrm{GeV} / c^{2}$ Higgs bosons produced through VBF, before and after $b$-jet energies are corrected. The mean shifts from $116 \mathrm{GeV} / c^{2}$ to $128 \mathrm{GeV} / c^{2}$ and the root mean square (RMS) from $15.6 \mathrm{GeV} / c^{2}$ to $13.7 \mathrm{GeV} / c^{2}$. The resolution, defined as the ratio between the RMS and the mean, shifts from 0.13 to 0.11 , an improvement of $18 \%$.

The $b$-jet energy corrections should be independent of the sample used to train and test the NN. The NN training and testing was repeated using $W H$ and $Z H$ events and similar results were obtained.

\section{Untagged jets neural network}

The angular distributions of untagged jets ( $q$ jets) from $V H$ or VBF differs from the angular distributions of generic jets contained within QCD multi-jet background events. Identification of $q$ jets can therefore help to separate signal events from QCD multi-jet background contributions. In particular, the $m_{q q}$ obtained from $q$ jets associated with the $W H$ and $Z H$ processes is constrained by the mass of the $W$ and $Z$, respectively. The $q$ jets produced in VBF events are typically separated by large $\phi$ and $\eta$, while the $q$ jets in QCD multi-jet events tend to exhibit a large difference in $\phi$ and a small difference in $\eta$. Three networks [24], referred to as $q q_{-} W H \mathrm{NN}, q q_{-} Z H \mathrm{NN}$, and $q q_{-} \mathrm{VBF} \mathrm{NN}$, are trained to separate events with $q$ jets originating from $W H, Z H$, and VBF production from background events. The input variables are $m_{q q}, \Delta \phi_{q q}, \Delta \eta_{q q}, \Delta R_{q q}$, and the transverse momenta of each $q$ jet with respect to the total momentum of the system. The networks are trained using Higgs MC to model signal and data-driven prediction for QCD multi-jets to model background. Examples of the output distributions of these trained neural network are shown in figure 7.

\footnotetext{
${ }^{5}$ The transverse mass is defined as $\left(p_{T} / p\right) M$, where $M$ is the invariant mass of the jet.

${ }^{6}$ The decay length is defined as the transverse distance between the primary vertex and the reconstructed secondary vertex in the SecVtx $b$-tagged jet.

${ }^{7}$ All NNs in this paper are trained using statistically independent samples.
} 


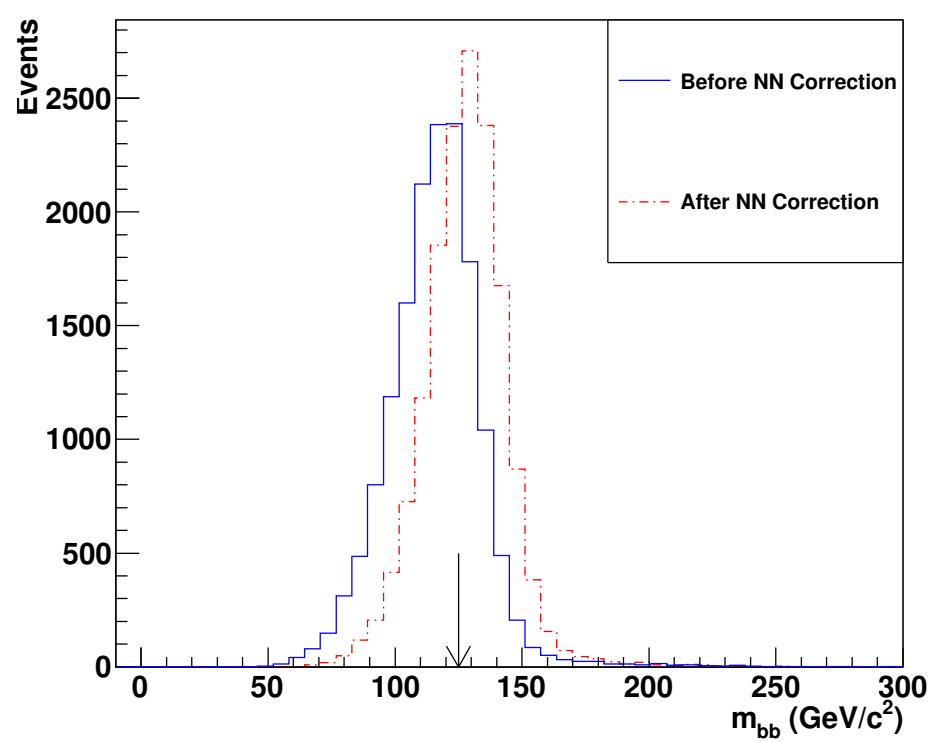

Figure 2. Comparison of $m_{b b}$ distribution in simulated decays of $125 \mathrm{GeV} / c^{2}$ Higgs bosons produced through VBF, before and after the $b$-jet energy correction for a VBF MC sample with $m_{H}=125 \mathrm{GeV} / c^{2}$ (indicated by the black arrow).

\section{Jet width}

The untagged jets ( $q$ jets) associated with the QCD multi-jet background are a mixture of quark and gluon jets whereas the $q$ jets associated with the Higgs signal are predominantly quark jets. As gluon jets tend to be broader than quark jets, jet width is another useful variable for distinguishing potential Higgs events from the background. We defined jet widths measured within the calorimeter $\left(\langle R\rangle_{\mathrm{CAL}}\right)$ and tracker $\left(\langle R\rangle_{\mathrm{TRK}}\right)$ as

$$
\begin{aligned}
\langle R\rangle_{\mathrm{CAL}} & =\sqrt{\sum_{\text {towers }}\left[\frac{E_{t}^{\text {tower }}}{E_{t}^{\text {jet }}}(\Delta R(\text { tower,jet }))^{2}\right]} \\
\langle R\rangle_{\mathrm{TRK}} & =\sqrt{\sum_{\text {tracks }}\left[\frac{P_{t}^{\text {track }}}{P_{t}^{\text {jet }}}(\Delta R(\text { track,jet }))^{2}\right]},
\end{aligned}
$$

where $\Delta R$ (tower,jet) ( $\Delta R$ (track,jet) $)$ is the angular distance between the jet axis and the calorimeter tower (track). All calorimeter towers within the jet cone of $\Delta R<0.4$ are used in the $\langle R\rangle_{\mathrm{CAL}}$ calculation. All tracks with $p_{T}>1 \mathrm{GeV} / c$ and within the jet cone of $\Delta R<0.4$ are used in the calculation of $\langle R\rangle_{\mathrm{TRK}}$.

The jet width also varies as a function of jet $E_{T}$, jet $\eta$, and the number of primary vertices $\left(N_{\mathrm{vtx}}\right)$, and is parameterized by a neural network fit. These dependences are removed by rescaling the measured jet widths to a common reference (that for a jet with $E_{T}=50 \mathrm{GeV}, \eta=0$, and $\left.N_{\mathrm{vtx}}=1\right)$ using the procedure described in ref. [11]. The NN function to parameterize the variation of jet width with jet $E_{T}$, jet $\eta$, and $N_{\mathrm{vtx}}$, is trained on a sample 
of untagged quark jets from the hadronic $W$ boson decays in $t \bar{t} \rightarrow b \bar{b} l \nu q q(\ell=e, \mu)$ events. The highest $E_{T}$ untagged-jet pair whose invariant mass is in the range $50-110 \mathrm{GeV} / c^{2}$ is assumed to originate from the hadronic $W$ boson decay. Separate networks are trained for $\mathrm{MC}$ and data. After rescaling, any differences in the jet width are assumed to be associated with the type of parton that initiated the jet. The $t \bar{t} \mathrm{MC}$ and data $q$-jet width distributions are found to agree after rescaling the measured jet widths. To check that the jet width rescaling can be applied to non-t $\bar{t}$ samples, the rescaling is also applied to the $q$ jets in $W H, Z H$, and $\mathrm{VBF} \mathrm{MC}$ events. The mean rescaled jet width in all samples is consistent with the width observed in the $t \bar{t}$ sample, which verifies the independence of the corrections with respect to jet $E_{T}, \eta$, and $N_{\mathrm{vtx}}$.

A systematic uncertainty is assigned by adding an offset to the rescaled $t \bar{t} \mathrm{MC}$ jet width and comparing the $\chi^{2} /$ degree of freedom ( $\chi^{2} /$ d.o.f $)$ of the shifted MC and $t \bar{t}$ data distributions with the unshifted MC and data. The uncertainty is defined by the offset that changes the $\chi^{2} /$ d.o.f by \pm 1 unit. The calorimeter jet width uncertainty is $\pm 2.6 \%$ and the tracker jet width uncertainty is $\pm 5.5 \%$.

Figures 4(c)-4(f) show the corrected jet width distributions of the untagged jets measured by the calorimeter and tracker. The Higgs signal tends to lower jet width values, which implies quark-like, whereas the QCD multi-jet tends to higher jet width, which implies a mixture of quark and gluons. The jet width distributions of the Higgs signal is different to the background which shows this variable is useful for the Higgs boson search.

\section{Classification of Higgs boson events}

A final NN is trained to optimize the separation of signal and background [24], which incorporates information from kinematic and angular jet variables, jet widths, event shapes, and the outputs of the untagged jets ( $q$ jets) NNs. The energies of the $b$ jets and widths of the $q$ jets are corrected as described in sections 7 and 9, respectively. As the $W H, Z H$, and VBF processes have different kinematics, dedicated $W H, Z H$, and VBF networks are trained separately for each process, resulting in three outputs. The outputs of the processspecific NNs are combined as inputs to a grand NN, referred to as the Higgs-NN. The output of the Higgs-NN is used to obtain Higgs search limits.

The selection of input variables for the process specific $W H, Z H$, and VBF networks training must fulfill two criteria: the variables must have good background-to-signal separation, and they must be well modeled by TRF. The discriminating variables for the $W H$-NN and $Z H$-NN training are $m_{b b}, m_{q q}$, the cosine of the leading-jet scattering angle in the four-jet rest-frame $\left(\cos \left(\theta_{3}\right)\right)$ [27], the $\chi$ variable $^{8}[11]$, the calorimeter jet width of the first $\left(\langle R\rangle_{\mathrm{CAL}}^{q_{1}}\right)$ and second leading untagged jet $\left(\langle R\rangle_{\mathrm{CAL}}^{q_{2}}\right)$, the tracker jet width of the first $\left(\langle R\rangle_{\mathrm{TRK}}^{q_{1}}\right)$ and second leading untagged jet $\left(\langle R\rangle_{\mathrm{TRK}}^{q_{2}}\right)$, aplanarity, ${ }^{9}$ sphericity, ${ }^{10}$ cen-

\footnotetext{
${ }^{8} \chi$ variable is the minimum of $\chi_{W}$ and $\chi_{Z}$ where $\chi_{W}=\sqrt{\left(M_{W}-m_{q q}\right)^{2}+\left(M_{H}-m_{b b}\right)^{2}}$ and a similar expression exists for $\chi_{z}$.

${ }^{9}$ Aplanarity measures the transverse momentum component out of the event plane.

${ }^{10}$ Sphericity is a measure of the summed transverse momentum squared with respect to the event axis.
} 
trality $^{11}[20], \Delta \mathrm{R}$ of the two $b$-tagged jets, $\Delta \mathrm{R}$ of the two untagged jets, $\Delta \phi$ of the two $b$-tagged jets, $\Delta \phi$ of the two untagged jets, and the $q q_{-} W H$ and $q q-Z H$ network outputs (section 8). Not all variables used in the $W H$ and $Z H$ networks' training have a good background-to-signal separation for VBF. For the VBF-NN training, the $\cos \left(\theta_{3}\right)$, the aplanarity, and the $\Delta \phi$ of the two untagged jets are removed; the $\eta$ angle of the first $\left(\eta_{q_{1}}\right)$ and second leading untagged jet $\left(\eta_{q_{2}}\right)$, the $\Delta \eta$ of the two untagged jets $\left(\Delta \eta_{q q}\right)$, the invariant mass of four jets system, the sum of the four jets' momenta along $z$ direction are added, and the $q q_{-} W H$ and $q q_{-} Z H$ network outputs are replaced by $q q_{-} \mathrm{VBF}$ NN output. Overall, the $V H(\mathrm{VBF})-\mathrm{NN}$ is trained with 17(18) variables, of which $m_{b b}$ and $m_{q q}\left(m_{q q}\right.$ and $\left.\Delta \eta_{q q}\right)$ are the most discriminating variables.

The distributions of the discriminating variables for the Higgs signal and background are shown in figures 3-7. The presence of a resonance in the $V H$ and VBF channels, due to the potential Higgs boson decay, produces a peak in the $m_{b b}$ that is not present in the QCD multi-jet background (figure 3(a)). A similar observation can be made for the $m_{q q}$ distribution in figure 3(b), where the $V H$ channel features a resonance due to the $W / Z$ decay (not observed in the VBF channel since the two $q$ jets are not produced from the decay of a particle). In figures 4(c)-(f) the jet width distributions of untagged jets of the QCD multi-jet background are broader than the Higgs signal due to the reason that is described in section 9. The two $q$ jets produced in the VBF events, which are produced from the two incoming quarks that each radiates a weak boson, tend to point in the forward but opposite directions. Thus the two $q$ jets are widely separated in the pseudo rapidity space. These features are shown in figures 5(a)-(c). The $q q_{-} W H$ NN (figure 7(a)), qq- $Z H$ NN (figure $7(\mathrm{~b})$ ), and $q q \mathrm{VBF}$ NN (figure $7(\mathrm{c})$ ) distributions are the outputs of three neural networks that are trained to separate the QCD multi-jet events from $\mathrm{WH}, \mathrm{ZH}$ and VBF productions, respectively.

Each variable demonstrates some ability to distinguish a Higgs boson from the background. Some variables, such as figures 3(c) and 7(a) appear to have some mismodeling of the background. However the observed difference are within the shape uncertainties of the TRF QCD multi-jet prediction. These shape uncertainties are derived by testing these variables in the TAG, CONTROL, and NJET6 control regions and propagating any difference as a shape uncertainty.

The $W H-\mathrm{NN}, Z H-\mathrm{NN}$, and VBF-NN are trained using dedicated $\mathrm{MC}$ samples for signal modeling. A small subset (10\%) of single-tagged jet events, after random selection and application of the TRF, is used as the QCD multi-jet training sample. The remaining $90 \%$ of events are reserved for modeling the NN output distributions. As the shapes of the kinematic distributions are found to be consistent for both $b$-tagging categories, the NN is trained using SS events.

The search focuses on Higgs boson mass hypotheses in the range $100 \leq m_{H} \leq$ $150 \mathrm{GeV} / c^{2}$ at $5 \mathrm{GeV} / c^{2}$ intervals. The sensitivity of the search is improved by using separate trainings at three specific Higgs boson masses: $100 \mathrm{GeV} / c^{2}, 120 \mathrm{GeV} / c^{2}$, and $140 \mathrm{GeV} / c^{2}$. For each Higgs boson mass hypothesis, we choose the training that gives the best search sensitivity.

\footnotetext{
${ }^{11}$ Centrality measures how much of the energy flows into the central rapidity region.
} 


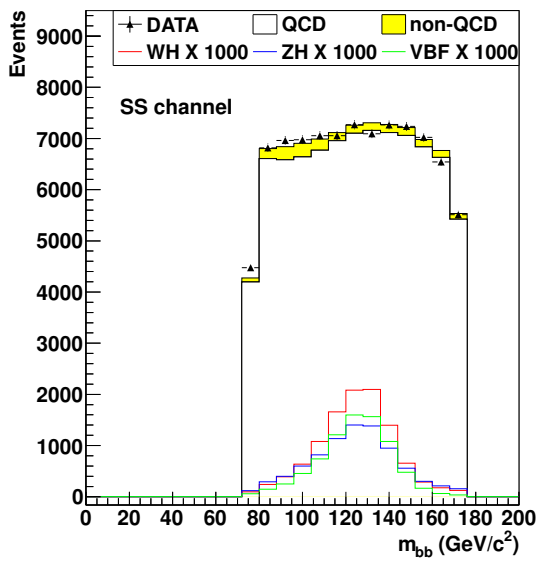

(a)

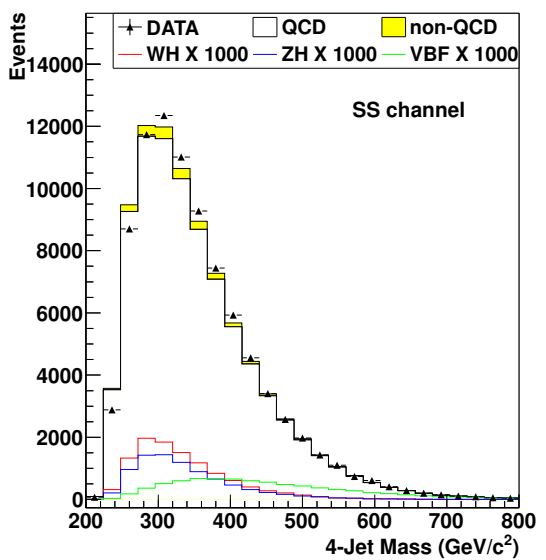

(c)

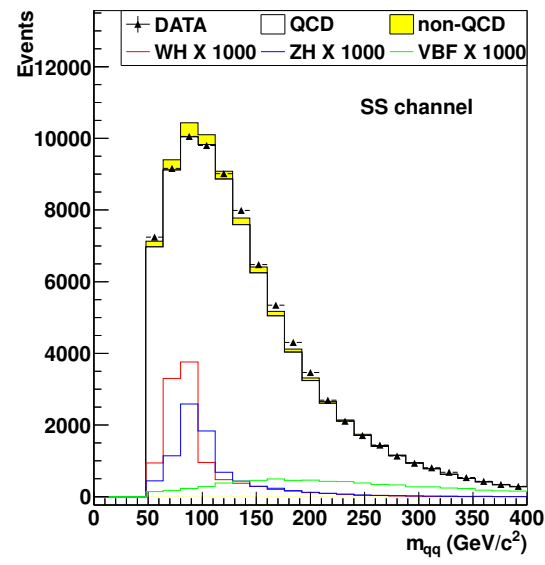

(b)

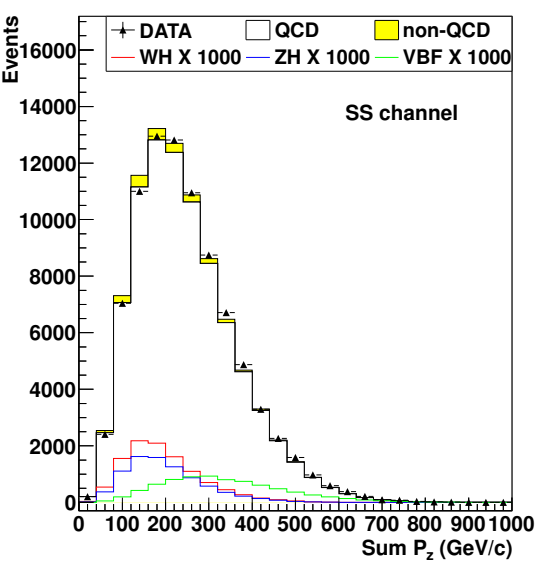

(d)

Figure 3. The QCD multi-jet background prediction (SS b-tag category) for (a) $m_{b b}$, (b) $m_{q q}$, (c) the invariant mass of four-jets system, and (d) the sum of the momenta along $z$ direction for each of the four jets in the search signal region. The $m_{q q}$ variable distribution is obtained after the application of the $m_{q q}$ correction described in section 6 . The black histograms are the TRF derived predictions for the QCD multi-jet background, and the black triangles are the data. The yellow histogram shows the MC predicted non-QCD background which is the sum of $t \bar{t}$, single-top, $Z+$ jets, $W+H F$, and diboson contributions. The predicted distributions for $W H$ events (red), $Z H$ events (blue), and VBF events (green) for a Higgs mass of $m_{H}=125 \mathrm{GeV} / c^{2}$ scaled by a factor of 1000 are also shown.

Only variables that are well modeled by the TRF are used to train the $W H-\mathrm{NN}$, $Z H-\mathrm{NN}$, and VBF-NN. As a further validation, the modeled outputs of the $W H, Z H$, and VBF networks are compared to TAG events in data. The $W H, Z H$ networks are found to be well modeled, but the VBF-NN requires an additional correction, analogous to the re-weighting performed to correct $m_{q q}$ (section 6 ). Figure 8 shows the Higgs-NN distribution of $125 \mathrm{GeV} / c^{2}$ Higgs boson events with both $b$ jets tagged by SecVtx, after 


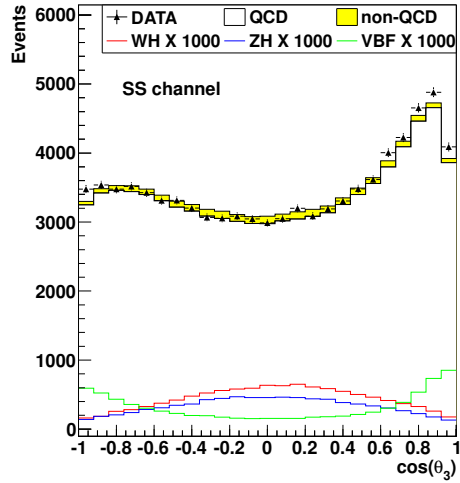

(a)

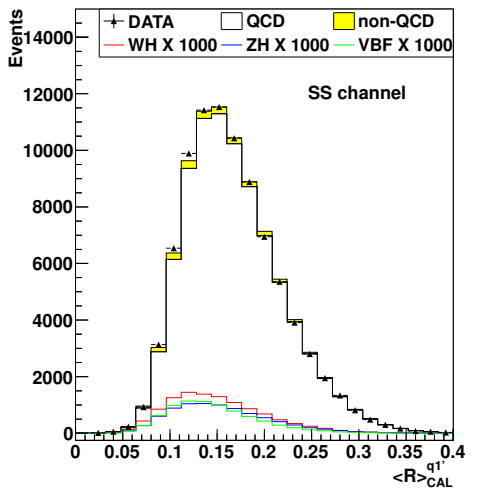

(c)

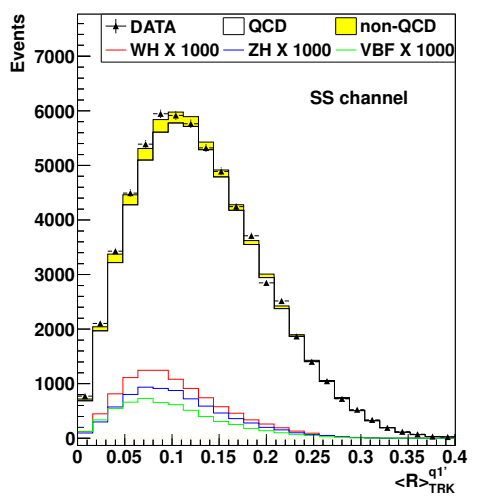

(e)

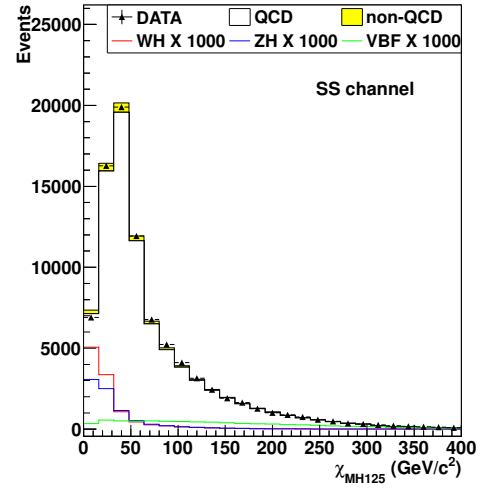

(b)

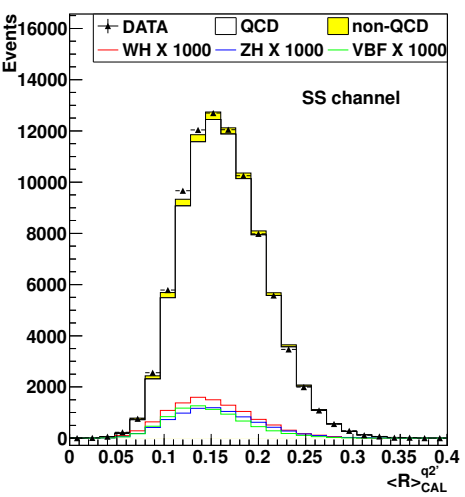

(d)

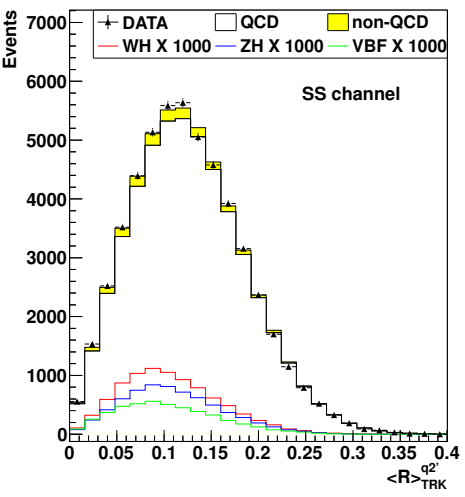

(f)

Figure 4. The QCD multi-jet background predictions for the SS b-tag category of (a) the cosine of the leading-jet scattering angle in the four-jet rest-frame [27], (b) the $\chi$ variable [11], (c) the calorimeter jet width of the first and (d) second leading untagged jet, and (e) the tracker jet width of the first and (f) second leading untagged jet. Descriptions of the signal and background histograms can be found in the caption of figure 3 . 


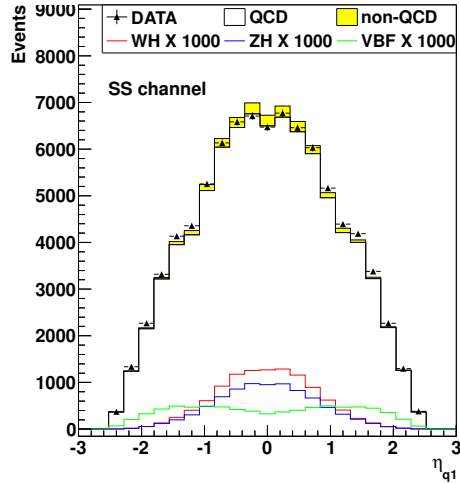

(a)

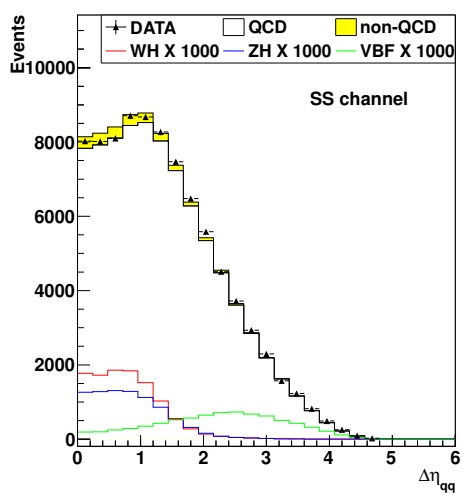

(c)

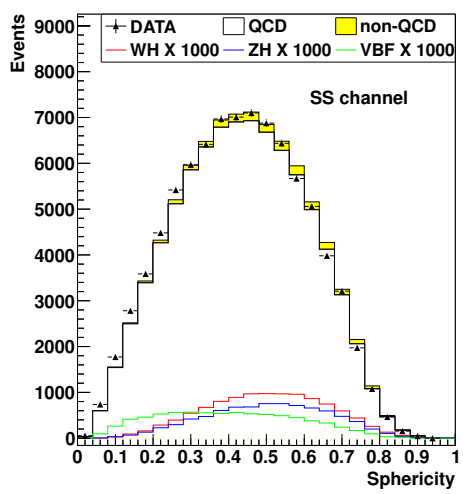

(e)

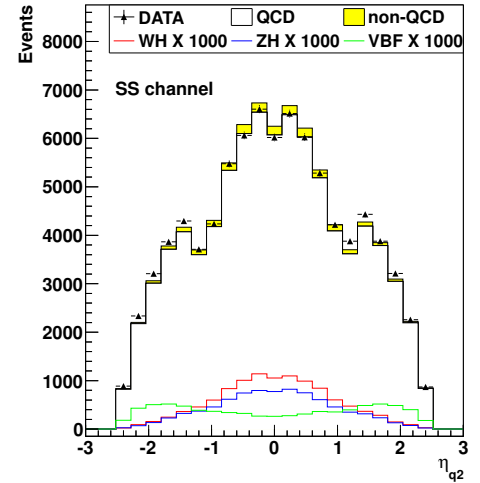

(b)

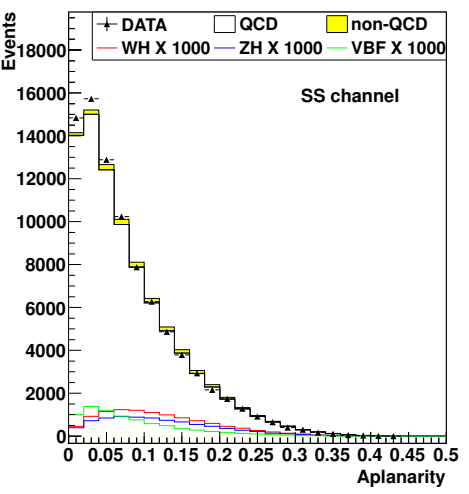

(d)

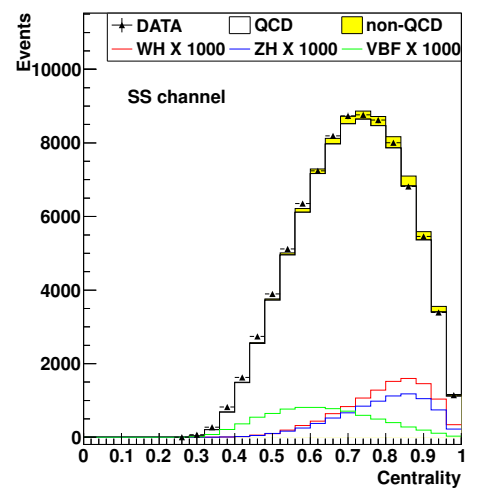

(f)

Figure 5. The QCD multi-jet background prediction for the SS $b$-tag category of (a) the $\eta$ angle of the first leading untagged jet and (b) second leading untagged jet, (c) $\Delta \eta$ of the two untagged jets, (d) the aplanarity [20], (e) the sphericity [20], and (f) centrality [20]. Descriptions of the signal and background histograms can be found in the caption of figure 3 . 


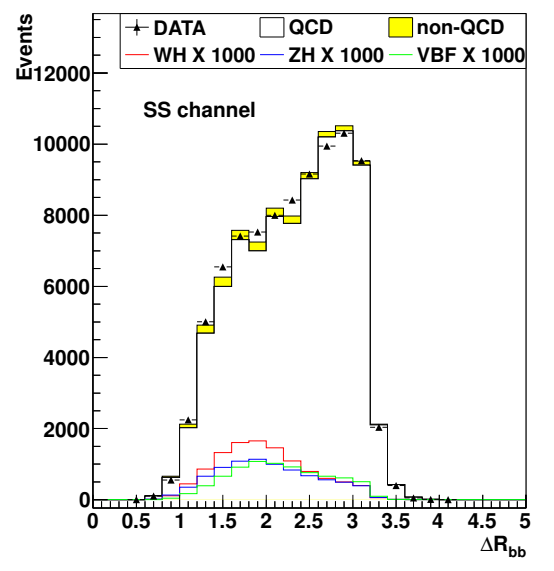

(a)

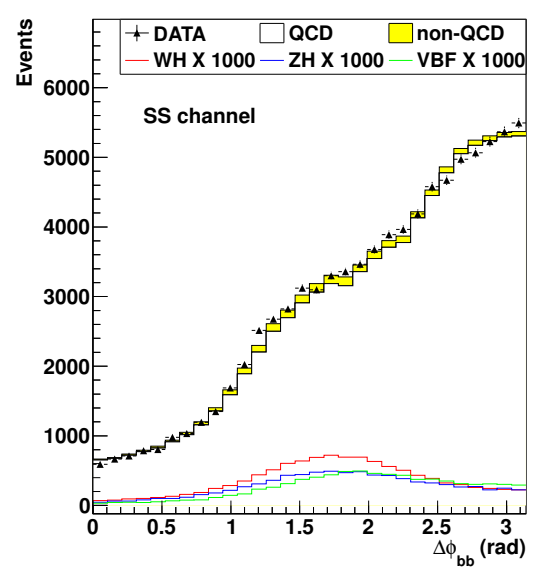

(c)

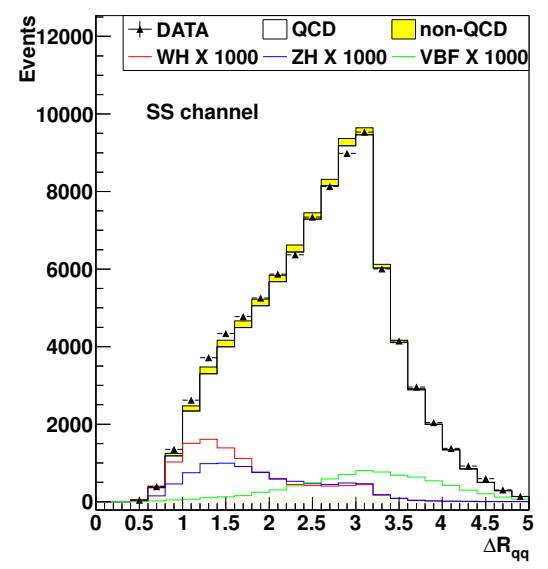

(b)

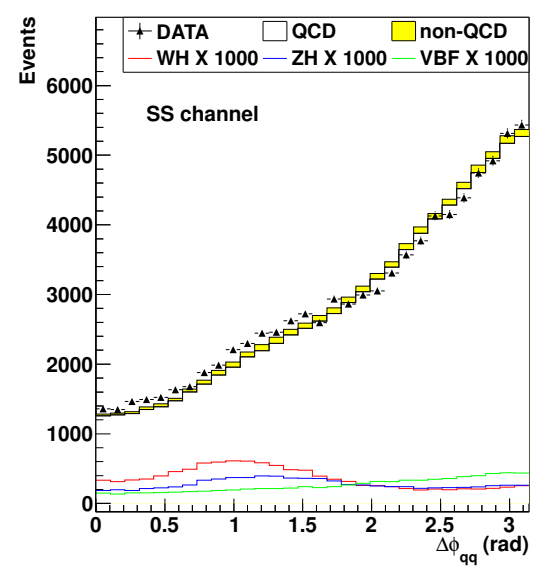

(d)

Figure 6. The QCD multi-jet background prediction for the SS b-tag category of (a) the $\Delta R$ of the two $b$-tagged jets and (b) of the two untagged jets, (c) the $\Delta \phi$ of the two $b$-tagged jets and (d) of the two untagged jets. Descriptions of the signal and background histograms can be found in the caption of figure 3 .

the VBF-NN correction function was applied. The histogram shows the data, a stacked distribution of the backgrounds, and the Higgs boson signal scaled by 1000. As the QCD multi-jet background is large, it is difficult to see the non-QCD contributions and the QCD uncertainty. In the lower QCD subtracted data plot, it is easier to see how well the background is modeled. This plot shows the QCD uncertainty is as large as the total non-QCD contributions and the QCD subtracted data is consistent with the non-QCD background and the QCD uncertainty. 


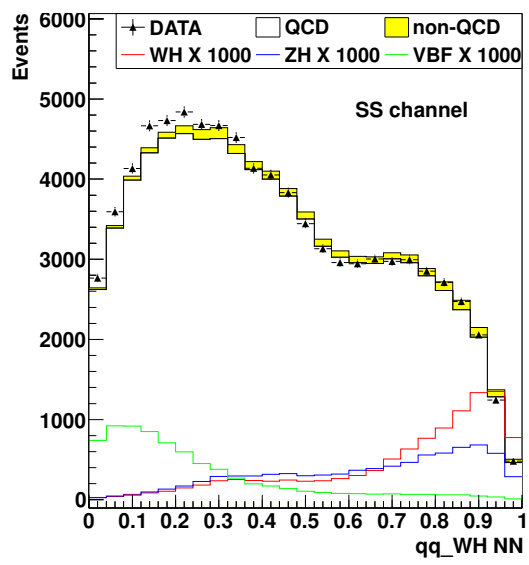

(a)

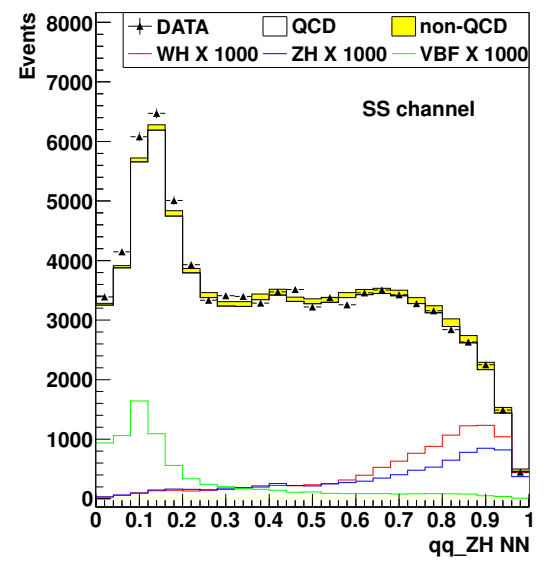

(b)

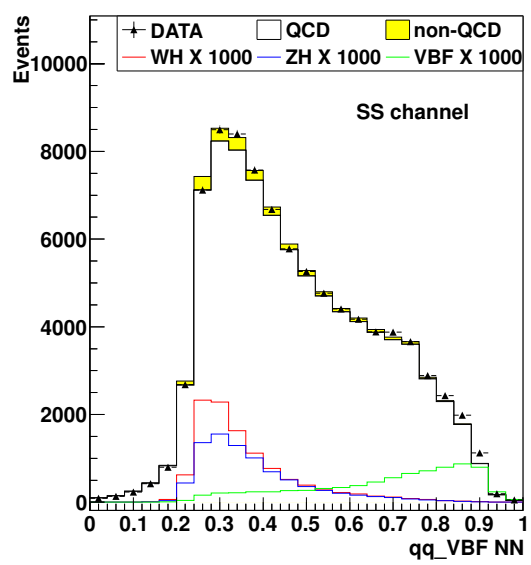

(c)

Figure 7. The QCD multi-jet background prediction for the SS $b$-tag category of the (a) $q q_{-} W H \mathrm{NN}$, (b) $q q_{-} Z H \mathrm{NN}$, and (c) $q q_{-} \mathrm{VBF} \mathrm{NN}$ (section 8). Descriptions of the signal and background histograms can be found in the caption of figure 3 .

\section{Systematic uncertainties}

This search considered systematic effects that affect the normalization (rate systematic uncertainty) and the output (shape systematic uncertainty) of the Higgs-NN for the signal and background. The rate systematic uncertainties are defined as the variations of the number of events that pass the selection requirements. The shape-related systematic uncertainties are expressed as fractional changes in the binned distributions.

The systematic effects that affect the normalization of the Higgs boson and nonQCD background are the uncertainty on the jet energy scale (JES) [17], on the PDF, $b$-tagging scale factor, initial and final state radiation (ISR and FSR), trigger efficiency, integrated luminosity, and cross section [5]. The effects that affect the shape of the Higgs boson and non-QCD backgrounds are the uncertainties on the JES, ISR, FSR, and the 


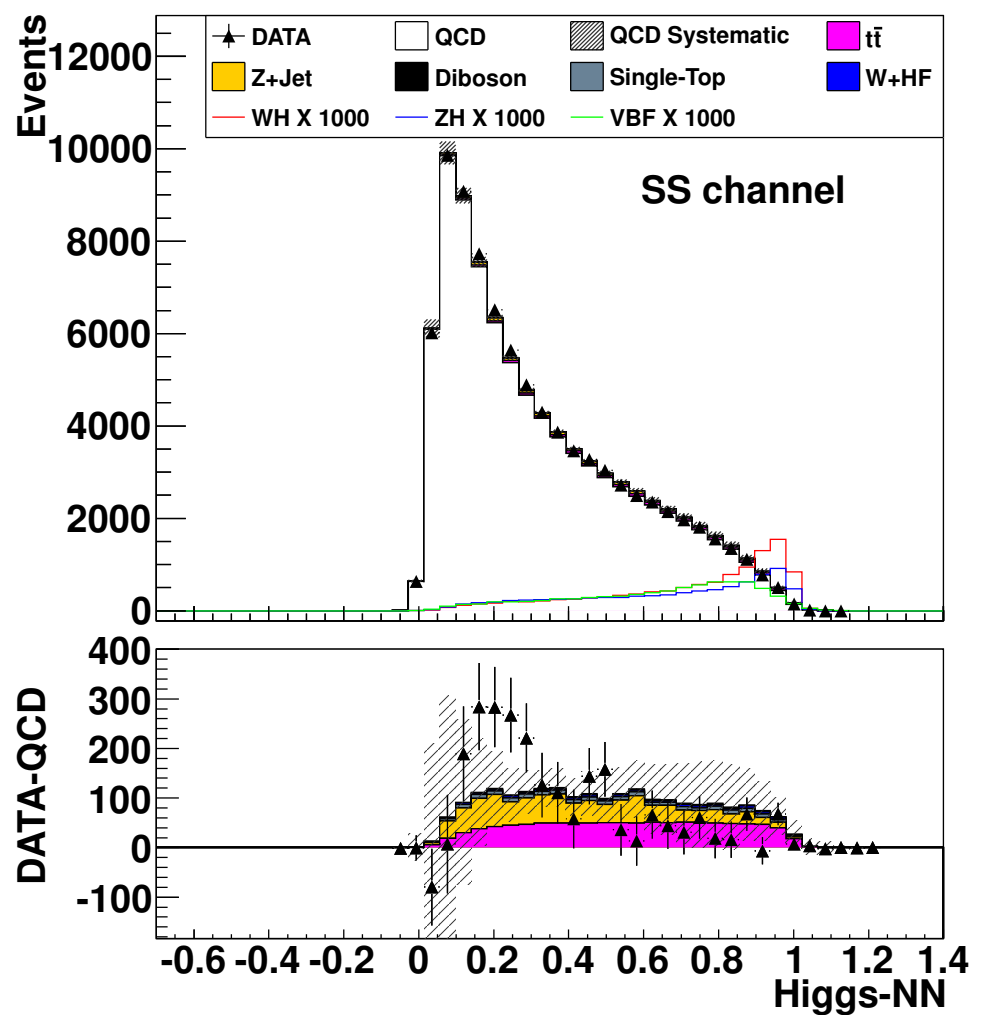

Figure 8. Higgs-NN distribution of $125 \mathrm{GeV} / c^{2}$ Higgs boson events with both $b$ jets tagged by SecVtx, after the VBF-NN correction function was applied. All backgrounds are stacked and the superimposed Higgs boson signal is scaled by 1000. As the QCD multi-jet background is large, plots of the difference of data and QCD multi-jet are plotted with a stacked plot of non-QCD background and QCD multi-jet systematic uncertainty. Both plots show the data are consistent with the background, especially at large Higgs-NN score where the higgs signal peaks.

jet width. The shape uncertainties are evaluated by adjusting their values by $\pm 1 \sigma$, and propagating this change through the event selection and Higgs-NN. Table 2 summarizes all systematic uncertainties.

Only shape uncertainties are considered for the QCD multi-jet component, the normalization is unconstrained. The TRF QCD shape uncertainties arise from uncertainties in the interpolation, $m_{q q}$ and VBF-NN correction functions. The TRF shape uncertainty is defined as the shape difference of the nominal QCD shape and a systematically shifted version.

The interpolation uncertainty accounts for sample-dependence of the TRF. A TRF is measured in the TAG region to its application in the signal region. Another TRF is measured in the CONTROL region (figure 1) and is applied to the signal region. The shape difference of the nominal TAG TRF and the CONTROL TRF defines the interpolation uncertainty.

The $m_{q q}$ and VBF-NN distributions require an additional correction to improve their TRF modeling (sections 6 and 10). The nominal correction functions are measured in 


\begin{tabular}{|c|c|}
\hline TRF (QCD multi-jet) $\mathrm{u}$ & rtainties \\
\hline TRF interpolation & Shape \\
\hline TRF $m_{q q}$ correction & Shape \\
\hline TRF VBF-NN correction & Shape \\
\hline Signal and Background un & ertainties \\
\hline Luminosity & $\pm 6 \%$ Rate \\
\hline Trigger & $\pm 3.55 \%$ Rate \\
\hline SecVtx + SecVtx & $\pm 7.1 \%$ Rate \\
\hline SecVtx+JetProb & $\pm 6.4 \%$ Rate \\
\hline Jet Energy Correction & $\pm 9 \%$ Rate \\
\hline & Shape \\
\hline Jet width & Shape \\
\hline Cross section uncertaintie & \\
\hline$t \bar{t}$ and single-top & $\pm 7 \%$ Rate \\
\hline Diboson (WW/WZ/ZZ) & $\pm 6 \%$ Rate \\
\hline$W+H F$ and $Z+$ jets & $\pm 50 \%$ Rate \\
\hline$W H / Z H$ & $\pm 5 \%$ Rate \\
\hline VBF & $\pm 10 \%$ Rate \\
\hline Signal uncertainties & \\
\hline $\mathrm{PDF}$ & $\pm 2 \%$ Rate \\
\hline ISR/FSR & $\pm 3 \%$ Rate \\
\hline & Shape \\
\hline
\end{tabular}

Table 2. Summary of all systematic uncertainties.

the TAG region and an alternative is measured in the CONTROL $\left(m_{q q}\right)$ and NJET6 (VBF-NN) regions. The shape difference between the usage of the nominal and alternative correction function defines the correction function shape uncertainty.

\section{Results}

The Higgs-NN output distribution in data is compared to the background predictions. No evidence of a Higgs boson signal is found, nor any disagreement between the predicted background and observed data. Upper exclusion limits are calculated on the Higgs boson cross-section at the $95 \% \mathrm{CL}$. The limits are calculated using a Bayesian method with a non-negative flat prior for the signal cross section. We integrate over Gaussian priors for the systematic uncertainties, truncated to ensure that no prediction is negative, and incorporate correlated rate and shape uncertainties as well as uncorrelated bin-by-bin statistical uncertainties [28]. Figure 9 and table 3 show the limits from the combination of SS and SJ $b$-tagging categories. The observed limits agree with the expected limits. 


\begin{tabular}{|c|l|l|l|l|l|l|}
\hline Higgs mass $\left(\mathrm{GeV} / c^{2}\right)$ & $-2 \sigma$ & $-1 \sigma$ & Median & $+1 \sigma$ & $+2 \sigma$ & Observed \\
\hline 100 & 1.4 & 3.6 & 7.7 & 14.5 & 24.4 & 10.9 \\
105 & 1.8 & 3.8 & 7.5 & 13.6 & 22.3 & 7.5 \\
110 & 2.0 & 4.0 & 7.6 & 13.2 & 21.7 & 7.0 \\
115 & 2.3 & 4.4 & 8.3 & 14.5 & 23.4 & 7.2 \\
120 & 2.4 & 4.6 & 8.9 & 15.6 & 25.3 & 8.4 \\
125 & 2.8 & 5.7 & 11.0 & 19.5 & 31.6 & 9.0 \\
130 & 3.4 & 7.1 & 13.8 & 24.3 & 39.5 & 13.2 \\
135 & 5.3 & 10.8 & 19.5 & 32.2 & 49.6 & 21.2 \\
140 & 7.3 & 14.3 & 25.8 & 42.7 & 66.1 & 26.2 \\
145 & 10.2 & 20.4 & 36.7 & 60.5 & 93.4 & 35.1 \\
150 & 17.1 & 32.5 & 58.7 & 98.2 & 152.0 & 64.6 \\
\hline
\end{tabular}

Table 3. Expected and observed 95\% CL upper limits for the combined SS and SJ channels. The limits are relative to the expected Higgs cross section.

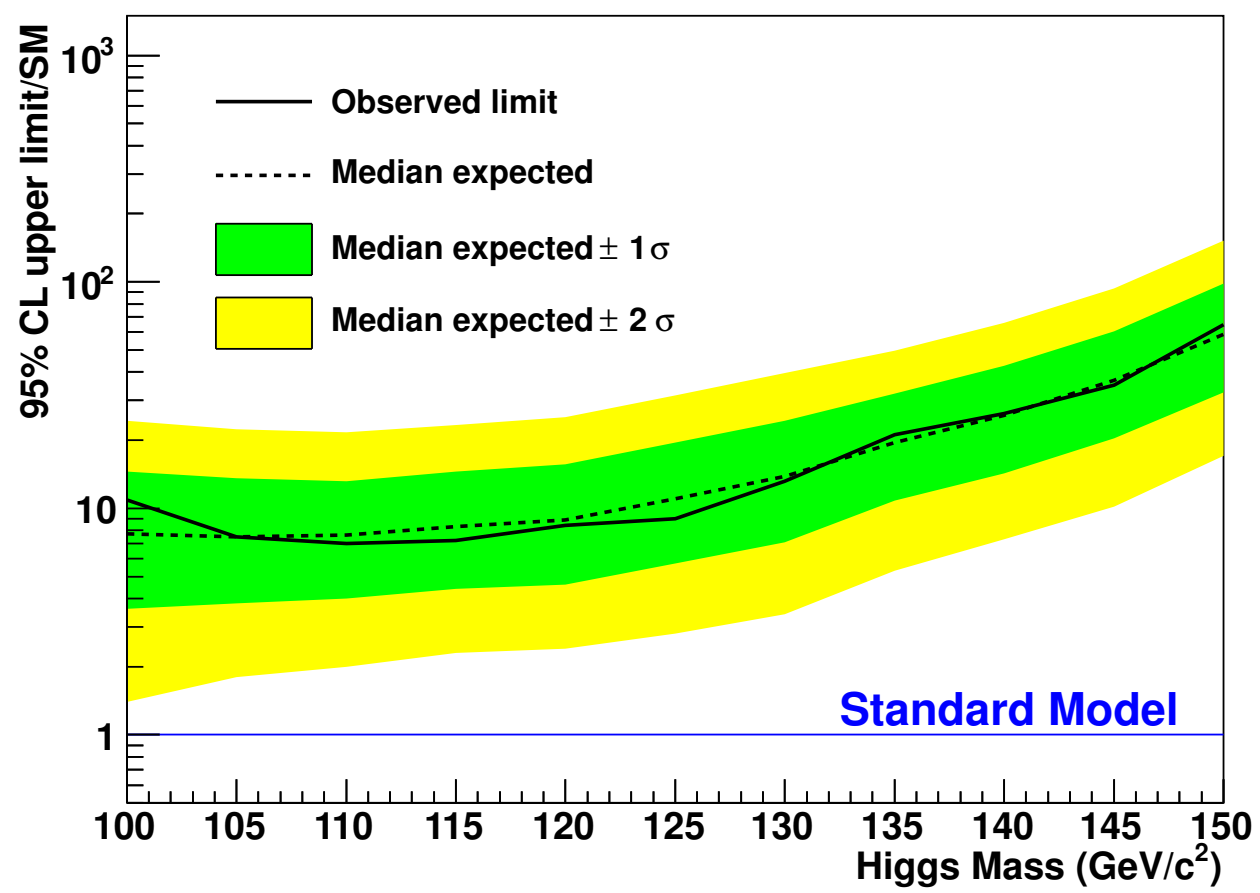

Figure 9. Upper limits at 95\% CL for combined SS and SJ channels: the expected and observed limits are plotted as a function of the Higgs boson mass. The limits are relative to the expected SM Higgs boson production, which includes the $H \rightarrow b \bar{b}$ branching ratio.

\section{Summary}

A search for the Higgs boson is performed in the all-hadronic final state using $9.45 \mathrm{fb}^{-1}$ of data collected by the CDF II detector. The results discussed in this paper have halved the 
expected limit of the previous search [11]. Half of the improvement comes from additional data and the expanded signal region contributes an additional 17\%. The reduction of the $b$-jet energy resolution by $18 \%$, adding a new jet width measurement, improving the QCD multi-jet modeling, and adding more variables in the Higgs neural network and improving its training contributes another $24 \%$. The combination of multi-variate techniques improved the best signal-to-background ratio from 0.0007 , if the $m_{b b}$ distribution alone was used for the search, to 0.006, which is almost a ten-fold increase. No significant Higgs boson signal is observed and upper exclusion limits are set on the observed Higgs cross section relative to the SM rate as a function of Higgs boson mass in the range $100-150 \mathrm{GeV} / c^{2}$. For a $125 \mathrm{GeV} / c^{2}$ Higgs boson, the 95\% CL expected (observed) limit is 11.0 (9.0) times the expected SM rate. This search is CDF's fourth most sensitive $H \rightarrow b \bar{b}$ search and is more sensitive than CDF's $t \bar{t} H$ [29] and similar to CDF's $H \rightarrow \gamma \gamma$ [30] searches, which have an expected limit of 12.6 and 9.9 for a $125 \mathrm{GeV} / c^{2}$ Higgs boson, respectively. CDF has also developed an improved algorithm to identify (tag) $b$-jets [31], which improves the $b$-tagging rate from $39 \%$ to $54 \%$ and was used in the latest $Z H \rightarrow \ell \ell b \bar{b}$ [32] and $W H \rightarrow \ell \nu b \bar{b}$ [33] searches. The addition of new $b$-jet tagger could potentially improve this search's expected limit by an additional $40 \%$ which would lower the expected limit to 7.9 times the expected $\mathrm{SM}$ rate for a $125 \mathrm{GeV} / c^{2}$ Higgs boson. The all-hadronic search is a unique channel at the Tevatron that has not been explored at the LHC. The improvements described in this paper, such as the data-driven QCD multi-jet prediction, $b$-jet energy corrections, jet width, and two-stage NN can be applied to $H \rightarrow b \bar{b}$ searches and other multi-jet analyses at the LHC.

\section{Acknowledgments}

We thank the Fermilab staff and the technical staffs of the participating institutions for their vital contributions. This work was supported by the U.S. Department of Energy and National Science Foundation; the Italian Istituto Nazionale di Fisica Nucleare; the Ministry of Education, Culture, Sports, Science and Technology of Japan; the Natural Sciences and Engineering Research Council of Canada; the National Science Council of the Republic of China; the Swiss National Science Foundation; the A.P. Sloan Foundation; the Bundesministerium für Bildung und Forschung, Germany; the Korean World Class University Program, the National Research Foundation of Korea; the Science and Technology Facilities Council and the Royal Society, U.K.; the Russian Foundation for Basic Research; the Ministerio de Ciencia e Innovación, and Programa Consolider-Ingenio 2010, Spain; the Slovak R\&D Agency; the Academy of Finland; and the Australian Research Council (ARC).

Open Access. This article is distributed under the terms of the Creative Commons Attribution License which permits any use, distribution and reproduction in any medium, provided the original author(s) and source are credited. 


\section{References}

[1] P.W. Higgs, Broken symmetries, massless particles and gauge fields, Phys. Lett. 12 (1964) 132 [InSPIRE].

[2] F. Englert and R. Brout, Broken symmetry and the mass of gauge vector mesons, Phys. Rev. Lett. 13 (1964) 321 [INSPIRE].

[3] P.W. Higgs, Broken symmetries and the masses of gauge bosons, Phys. Rev. Lett. 13 (1964) 508 [InSPIRE].

[4] LeP Working Group for Higgs boson searches, AlEPH, DELPHi, L3, OPAL collaborations, R. Barate et al., Search for the standard model Higgs boson at LEP, Phys. Lett. B 565 (2003) 61 [hep-ex/0306033] [INSPIRE].

[5] Tevatron New Physics Higgs Working Group, CDF, D0 collaborations, Updated combination of CDF and DO searches for standard model Higgs boson production with up to 10.0 $\mathrm{fb}^{-1}$ of data, arXiv: 1207.0449 [INSPIRE].

[6] CMS collaboration, Observation of a new boson at a mass of $125 \mathrm{GeV}$ with the CMS experiment at the LHC, Phys. Lett. B 716 (2012) 30 [arXiv:1207.7235] [INSPIRE].

[7] ATLAS collaboration, Observation of a new particle in the search for the standard model Higgs boson with the ATLAS detector at the LHC, Phys. Lett. B 716 (2012) 1 [arXiv: 1207.7214] [INSPIRE].

[8] CDF, D0 collaborations, Evidence for a particle produced in association with weak bosons and decaying to a bottom-antibottom quark pair in Higgs boson searches at the Tevatron, Phys. Rev. Lett. 109 (2012) 071804 [arXiv: 1207.6436] [INSPIRE].

[9] A. Djouadi, J. Kalinowski and M. Spira, HDECAY: a program for Higgs boson decays in the standard model and its supersymmetric extension, Comput. Phys. Commun. 108 (1998) 56 [hep-ph/9704448] [INSPIRE].

[10] CDF collaboration, A search for the associated production of the standard-model Higgs boson in the all-hadronic channel, Phys. Rev. Lett. 103 (2009) 221801 [arXiv:0907.0810] [INSPIRE].

[11] CDF collaboration, Search for the Higgs boson in the all-hadronic final state using the CDF II detector, Phys. Rev. D 84 (2011) 052010 [arXiv:1102.0024] [InSPIRE].

[12] CDF collaboration, Search for Higgs bosons produced in association with a vector boson in $p \bar{p}$ collisions at $\sqrt{s}=1.8 \mathrm{TeV}$, Phys. Rev. Lett. 81 (1998) 5748 [INSPIRE].

[13] CDF collaboration, Measurement of the $J / \psi$ meson and b-hadron production cross sections in $p \bar{p}$ collisions at $\sqrt{s}=1960$ GeV, Phys. Rev. D 71 (2005) 032001 [hep-ex/0412071] [INSPIRE].

[14] CDF collaboration, Measurement of the $t \bar{t}$ production cross section in p $\bar{p}$ collisions at $\sqrt{s}=1.96$ TeV using lepton + jets events with secondary vertex b-tagging, Phys. Rev. D 71 (2005) 052003 [hep-ex/0410041] [InSPIRE].

[15] CDF collaboration, Measurements of inclusive $W$ and $Z$ cross sections in $p \bar{p}$ collisions at $\sqrt{s}=1.96$ TeV, J. Phys. G 34 (2007) 2457 [hep-ex/0508029] [InSPIRE].

[16] CDF collaboration, The topology of three jet events in $\bar{p} p$ collisions at $\sqrt{s}=1.8$ TeV, Phys. Rev. D 45 (1992) 1448 [INSPIRE]. 
[17] CDF collaboration, Determination of the jet energy scale at the collider detector at Fermilab, Nucl. Instrum. Meth. A 566 (2006) 375 [hep-ex/0510047] [INSPIRE].

[18] CDF collaboration, Measurement of the $t \bar{t}$ production cross section in $p \bar{p}$ collisions at $\sqrt{s}=1.96$ TeV using lepton + jets events with jet probability $B^{-}$tagging, Phys. Rev. D 74 (2006) 072006 [hep-ex/0607035] [InSPIRE].

[19] M.L. Mangano, M. Moretti, F. Piccinini, R. Pittau and A.D. Polosa, ALPGEN, a generator for hard multiparton processes in hadronic collisions, JHEP 07 (2003) 001 [hep-ph/0206293] [INSPIRE].

[20] T. Sjöstrand, L. Lönnblad and S. Mrenna, PYTHIA 6.2: physics and manual, hep-ph/0108264 [INSPIRE].

[21] CTEQ collaboration, H. Lai et al., Global QCD analysis of parton structure of the nucleon: CTEQ5 parton distributions, Eur. Phys. J. C 12 (2000) 375 [hep-ph/9903282] [INSPIRE].

[22] E. Gerchtein and M. Paulini, CDF detector simulation framework and performance, eConf $\mathbf{C}$ 0303241 (2003) TUMT005 [physics/0306031] [INSPIRE].

[23] R. Brun et al., GEANT 3: user's guide for Geant 3.10, Geant 3.11, CERN-DD-EE-84-01 (1987).

[24] A. Hocker, J. Stelzer, F. Tegenfeldt, H. Voss, K. Voss, et al., TMVA - Toolkit for multivariate data analysis, PoS ACAT (2007) 040 [physics/0703039] [INSPIRE].

[25] R. Brun and F. Rademakers, ROOT - An object oriented data analysis framework, Nucl. Inst. and Math. in Phys. Res. A 389 (1997) 81.

[26] T. Aaltonen, A. Buzatu, B. Kilminster, Y. Nagai and W. Yao, Improved b-jet energy correction for $H \rightarrow b \bar{b}$ searches at $C D F$, arXiv:1107.3026 [INSPIRE].

[27] S. Geer and T. Asakawa, The analysis of multi - Jet events produced at high-energy hadron colliders, Phys. Rev. D 53 (1996) 4793 [hep-ph/9510351] [InSPIRE].

[28] CDF, D0 collaboration, Combination of Tevatron searches for the standard model Higgs boson in the $w^{+} w^{-}$decay mode, Phys. Rev. Lett. 104 (2010) 061802 [arXiv:1001.4162] [INSPIRE].

[29] CDF collaboration, Search for the standard model Higgs boson produced in association with top quarks using the full CDF data set, Phys. Rev. Lett. 109 (2012) 181802 [arXiv: 1208.2662] [INSPIRE].

[30] CDF collaboration, Search for a Higgs boson in the diphoton final state using the full CDF data set from proton-antiproton collisions at $\sqrt{s}=1.96$ TeV, Phys. Lett. B 717 (2012) 173 [arXiv:1207.6386] [INSPIRE].

[31] J. Freeman, T. Junk, M. Kirby, Y. Oksuzian, T. Phillips, et al., Introduction to HOBIT, a b-jet identification tagger at the CDF experiment optimized for light Higgs boson searches, Nucl. Instrum. Meth. A 697 (2013) 64 [arXiv:1205.1812] [INSPIRE].

[32] CDF collaboration, Search for the standard model Higgs boson decaying to a bb pair in events with two oppositely-charged leptons using the full CDF data set, Phys. Rev. Lett. 109 (2012) 111803 [arXiv:1207.1704] [INSPIRE].

[33] CDF collaboration, Search for the standard model Higgs boson decaying to a bb pair in events with one charged lepton and large missing transverse energy using the full CDF data set, Phys. Rev. Lett. 109 (2012) 111804 [arXiv:1207.1703] [INSPIRE]. 


\section{The CDF collaboration}

T. Aaltonen ${ }^{21}$, B. Álvarez González ${ }^{9}$, S. Amerio ${ }^{40}$, D. Amidei ${ }^{32}$, A. Anastassov ${ }^{15}$, A. Annovi ${ }^{17}$, J. Antos ${ }^{12}$, G. Apollinari ${ }^{15}$, J.A. Appel ${ }^{15}$, A. Apresyan ${ }^{44, j j}$, T. Arisawa ${ }^{54}$, A. Artikov ${ }^{13}$, J. Asaadi ${ }^{49}$, W. Ashmanskas ${ }^{15}$, B. Auerbach ${ }^{57}$, A. Aurisano ${ }^{49}$, F. Azfar ${ }^{39}$, W. Badgett ${ }^{15}$, T. Bae ${ }^{25}$, A. BarbaroGaltieri $^{26}$, V.E. Barnes ${ }^{44}$, B.A. Barnett ${ }^{23}$, P. Barria ${ }^{42, \text { hh }}$, P. Bartos ${ }^{12}$, M. Bauce ${ }^{40, f f}$, F. Bedeschi ${ }^{42}$, S. Behari ${ }^{23}$, G. Bellettini ${ }^{42, g g}$, J. Bellinger ${ }^{56}$, D. Benjamin ${ }^{14}$, A. Beretvas ${ }^{15}$, A. Bhatti ${ }^{46}$, D. Bisello ${ }^{40, f f}$, I. Bizjak ${ }^{28}$, K.R. Bland ${ }^{5}$, B. Blumenfeld ${ }^{23}$, A. Bocci ${ }^{14}$, A. Bodek $^{45}$, D. Bortoletto ${ }^{44}$, J. Boudreau ${ }^{43}$, A. Boveia ${ }^{11}$, L. Brigliadori ${ }^{6, e e}$, C. Bromberg ${ }^{33}$, E. Brucken ${ }^{21}$, J. Budagov ${ }^{13}$, H.S. Budd ${ }^{45}$, K. Burkett ${ }^{15}$, G. Busetto ${ }^{40, f f}$, P. Bussey ${ }^{19}$, A. Buzatu $^{31}$, A. Calamba ${ }^{10}$, C. Calancha ${ }^{29}$, S. Camarda ${ }^{4}$, M. Campanelli28, M. Campbell ${ }^{32}$, F. Canelli11,15, B. Carls ${ }^{22}$, D. Carlsmith ${ }^{56}$,

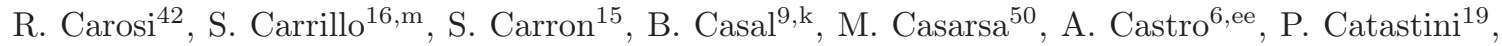
D. Cauz $^{50}$, V. Cavaliere ${ }^{22}$, M. Cavalli-Sforza ${ }^{4}$, A. Cerri ${ }^{26, f}$, L. Cerrito ${ }^{28, s}$, Y.C. Chen ${ }^{1}$, M. Chertok ${ }^{7}$, G. Chiarelli ${ }^{42}$, G. Chlachidze $^{15}$, F. Chlebana ${ }^{15}$, K. Cho $^{25}$, D. Chokheli ${ }^{13}$, W.H. Chung ${ }^{56}$, Y.S. Chung ${ }^{45}$, M.A. Ciocci ${ }^{42, h h}$, A. Clark $^{18}$, C. Clarke ${ }^{55}$, G. Compostella ${ }^{40, f f}$, M.E. Convery ${ }^{15}$, J. Conway ${ }^{7}$, M.Corbo ${ }^{15}$, M. Cordelli ${ }^{17}$, C.A. Cox ${ }^{7}$, D.J. Cox ${ }^{7}$, F. Crescioli ${ }^{42, g g}$, J. Cuevas ${ }^{9, z}$, R. Culbertson ${ }^{15}$, D. Dagenhart ${ }^{15}$, N. d'Ascenzo ${ }^{15, w}$, M. Datta ${ }^{15}$, P. de Barbaro ${ }^{45}$, M. Dell'Orso ${ }^{42, g g}$, L. Demortier ${ }^{46}$, M. Deninno ${ }^{6}$, F. Devoto ${ }^{21}$, M. d'Errico ${ }^{40, f f}$, A. Di Canto ${ }^{42, g g}$, B. Di Ruzza ${ }^{15}$, J.R. Dittmann ${ }^{5}$, M. D'Onofrio ${ }^{27}$, S. Donati ${ }^{42, g g}$, P. Dong ${ }^{15}$, M. Dorigo ${ }^{50}$, T. Dorigo ${ }^{40}$, K. Ebina ${ }^{54}$, A. Elagin ${ }^{49}$, A. Eppig ${ }^{32}$, R. Erbacher ${ }^{7}$, S. Errede ${ }^{22}$, N. Ershaidat ${ }^{15, d d}$, R. Eusebi ${ }^{49}$, S. Farrington ${ }^{39}$, M. Feindt ${ }^{24}$, J.P. Fernandez ${ }^{29}$, R. Field ${ }^{16}$, G. Flanagan ${ }^{15, u}$, R. Forrest ${ }^{7}$, M.J. Frank ${ }^{5}$, M. Franklin ${ }^{19}$, J.C. Freeman ${ }^{15}$, Y. Funakoshi ${ }^{54}$, I. Furic ${ }^{16}$, M. Gallinaro ${ }^{46}$, J.E. Garcia ${ }^{18}$, A.F. Garfinkel ${ }^{44}$, P. Garosi ${ }^{42, \text { hh }}$, H. Gerberich ${ }^{22}$, E. Gerchtein ${ }^{15}$, S. Giagu ${ }^{47}$, V. Giakoumopoulou ${ }^{3}$, P. Giannetti ${ }^{42}$, K. Gibson ${ }^{43}$, C.M. Ginsburg ${ }^{15}$, N. Giokaris ${ }^{3}$, P. Giromini ${ }^{17}$, G. Giurgiu ${ }^{23}$, V. Glagolev ${ }^{13}$, D. Glenzinski ${ }^{15}$, M. Gold ${ }^{35}$, D. Goldin ${ }^{49}$, N. Goldschmidt ${ }^{16}$, A. Golossanov ${ }^{15}$, G. Gomez ${ }^{9}$, G. Gomez-Ceballos ${ }^{30}$, M. Goncharov ${ }^{30}$, O. González ${ }^{29}$, I. Gorelov ${ }^{35}$, A.T. Goshaw ${ }^{14}$, K. Goulianos ${ }^{46}$, S. Grinstein ${ }^{4}$, C. Grosso-Pilcher ${ }^{11}$, R.C. Group ${ }^{15,53}$, J. Guimaraes da Costa $^{19}$, S.R. $\operatorname{Hahn}^{15}$, E. Halkiadakis ${ }^{48}$, A. Hamaguchi ${ }^{38}$, J.Y. Han ${ }^{45}$, F. Happacher ${ }^{17}$, K. Hara ${ }^{51}$, D. Hare ${ }^{48}$, M. Hare ${ }^{52}$, R.F. Harr ${ }^{55}$, K. Hatakeyama ${ }^{5}$, C. Hays ${ }^{39}$, M. Heck ${ }^{24}$, J. Heinrich ${ }^{41}$, M. Herndon ${ }^{56}$, S. Hewamanage ${ }^{5}$, A. Hocker ${ }^{15}$, W. Hopkins ${ }^{15, g}$, D. Horn ${ }^{24}$, S. Hou ${ }^{1}$, R.E. Hughes ${ }^{36}$, M. Hurwitz ${ }^{11}$, U. Husemann ${ }^{57}$, N. Hussain ${ }^{31}$, M. Hussein ${ }^{33}$, J. Huston ${ }^{33}$, G. Introzzi ${ }^{42, k k}$, M. Iori ${ }^{47, j j}$, A. Ivanov ${ }^{7, p}$, E. James ${ }^{15}$, D. Jang ${ }^{10}$, B. Jayatilaka ${ }^{14}$, E.J. Jeon ${ }^{25}$, S. Jindariani ${ }^{15}$, M. Jones ${ }^{44}$, K.K. Joo ${ }^{25}$, S.Y. Jun ${ }^{10}$, T.R. Junk ${ }^{15}$, T. Kamon ${ }^{49,25}$, P.E. Karchin ${ }^{55}$, A. Kasmi ${ }^{5}$, Y. Kato ${ }^{38, \text { o }}$, W. Ketchum ${ }^{11}$, J. Keung ${ }^{41}$, V. Khotilovich ${ }^{49}$, B. Kilminster ${ }^{15}$, D.H. Kim ${ }^{25}$, H.S. Kim ${ }^{25}$, J.E. $\mathrm{Kim}^{25}$, M.J. Kim${ }^{17}$, S.B. Kim${ }^{25}$, S.H. Kim${ }^{51}$, Y.K. Kim${ }^{11}$, Y.J. Kim${ }^{25}$, N. Kimura ${ }^{54}$, M. Kirby ${ }^{15}$, S. Klimenko ${ }^{16}$, K. Knoepfel ${ }^{15}$, K. Kondo ${ }^{1254}$, D.J. Kong ${ }^{25}$, J. Konigsberg ${ }^{16}$, A.V. Kotwal ${ }^{14}$, M. Kreps ${ }^{24}$, J. Kroll ${ }^{41}$, D. Krop ${ }^{11}$, M. Kruse ${ }^{14}$, V. Krutelyov ${ }^{49, c}$, T. Kuhr ${ }^{24}$, M. Kurata ${ }^{51}$, S. Kwang ${ }^{11}$, A.T. Laasanen ${ }^{44}$, S. Lami ${ }^{42, g g, h h, i i}$, S. Lammel ${ }^{15}$, M. Lancaster ${ }^{28}$, R.L. Lander ${ }^{7}$, K. Lannon ${ }^{36, y}$, A. Lath $^{48}$, G. Latino ${ }^{42, \text { hh }}$, T. LeCompte ${ }^{2}$, E. Lee ${ }^{49}$, H.S. Lee ${ }^{11, q}$, J.S. $\mathrm{Lee}^{25}$, S.W. Lee ${ }^{49, \mathrm{bb}}$, S. Leo ${ }^{42, \mathrm{gg}}$, S. Leone ${ }^{42}$, J.D. Lewis ${ }^{15}$, A. Limosani ${ }^{14, t}$, C.-J. Lin ${ }^{26}$,

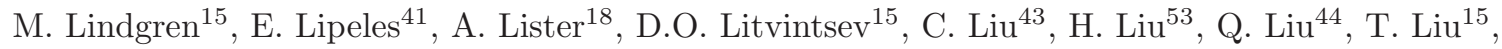
S. Lockwitz ${ }^{57}$, A. Loginov ${ }^{57}$, D. Lucchesi ${ }^{40, f f}$, J. Lueck ${ }^{24}$, P. Lujan ${ }^{26}$, P. Lukens ${ }^{15}$, G. Lungu ${ }^{46}$, J. Lys ${ }^{26}$, R. Lysak ${ }^{12, \text { e }}$, R. Madrak ${ }^{15}$, K. Maeshima ${ }^{15}$, P. Maestro ${ }^{42, h h}$, S. Malik ${ }^{46}$, G. Manca ${ }^{27, a}$, A. Manousakis-Katsikakis ${ }^{3}$, F. Margaroli47 , C. Marino ${ }^{24}$, M. Martínez ${ }^{4}$, P. Mastrandrea ${ }^{47}$, K. Matera ${ }^{22}$, M.E. Mattson ${ }^{55}$, A. Mazzacane ${ }^{15}$, P. Mazzanti ${ }^{6}$, K.S. McFarland ${ }^{45}$, P. McIntyre $^{49}$, R. McNulty 27, , A. Mehta ${ }^{27}$, P. Mehtala ${ }^{21}$, C. Mesropian $^{46}$, T. Miao ${ }^{15}$, D. Mietlicki ${ }^{32}$, A. Mitra ${ }^{1}$, H. Miyake ${ }^{51}$, S. Moed ${ }^{15}$, N. Moggi ${ }^{6}$, M.N. Mondragon ${ }^{15, m}$, C.S. Moon ${ }^{25}$, R. Moore ${ }^{15}$,

\footnotetext{
${ }^{12}$ Deceased
} 
M.J. Morello ${ }^{42, i i}$, J. Morlock ${ }^{24}$, P. Movilla Fernandez ${ }^{15}$, A. Mukherjee ${ }^{15}$, Th. Muller ${ }^{24}$, P. Murat ${ }^{15}$, M. Mussini 6 ,ee, J. Nachtman ${ }^{15, n}$, Y. Nagai ${ }^{51}$, J. Naganoma ${ }^{54}$, I. Nakano ${ }^{37}$, A. Napier ${ }^{52}$, J. Nett ${ }^{49}$, C. Neu ${ }^{53}$, M.S. Neubauer ${ }^{22}$, J. Nielsen ${ }^{26, d}$, L. Nodulman ${ }^{2}$, S.Y. Noh ${ }^{25}$, O. Norniella ${ }^{22}$, L. Oakes ${ }^{39}$, S.H. Oh ${ }^{14}$, Y.D. Oh ${ }^{25}$, I. Oksuzian ${ }^{53}$, T. Okusawa ${ }^{38}$, R. Orava ${ }^{21}$, L. Ortolan ${ }^{4}$, S. Pagan Griso ${ }^{40, f f}$, C. Pagliarone ${ }^{50}$, E. Palencia ${ }^{9, f}$, V. Papadimitriou ${ }^{15}$, A.A. Paramonov ${ }^{2}$, J. Patrick ${ }^{15}$, G. Pauletta ${ }^{50, k k}$, M. Paulini ${ }^{10}$, C. Paus ${ }^{30}$, D.E. Pellett ${ }^{7}$, A. Penzo ${ }^{50}$, T.J. Phillips ${ }^{14}$, G. Piacentino ${ }^{42}$, E. Pianori ${ }^{41}$, J. Pilot ${ }^{36}$, K. Pitts ${ }^{22}$, C. Plager ${ }^{8}$, L. Pondrom ${ }^{56}$, S. Poprocki ${ }^{15, g}$,

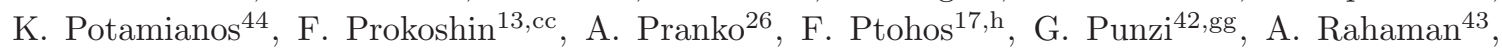
V. Ramakrishnan ${ }^{56}$, N. $\operatorname{Ranjan}^{44}$, I. Redondo ${ }^{29}$, P. Renton ${ }^{39}$, M. Rescigno ${ }^{47}$, T. Riddick ${ }^{28}$, F. Rimondi ${ }^{6, e e}$, L. Ristori ${ }^{15,42}$, A. Robson ${ }^{19}$, T. Rodrigo ${ }^{9}$, T. Rodriguez ${ }^{41}$, E. Rogers $^{22}$, S. Rolli $^{52, i}$, R. $\operatorname{Roser}^{15}$, F. Ruffini ${ }^{42, \text { hh }}$, A. Ruiz ${ }^{9}$, J. Russ ${ }^{10}$, V. Rusu ${ }^{15}$, A. Safonov ${ }^{49}$, W.K. Sakumoto ${ }^{45}$, Y. Sakurai ${ }^{54}$, L. Santi ${ }^{50, k k}$, K. Sato ${ }^{51}$, V. Saveliev ${ }^{15, w}$, A. Savoy-Navarro ${ }^{15, a a}$, P. Schlabach ${ }^{15}$, A. Schmidt ${ }^{24}$, E.E. Schmidt ${ }^{15}$, T. Schwarz ${ }^{15}$, L. Scodellaro ${ }^{9}$, A. Scribano ${ }^{42, h h}$, F. Scuri ${ }^{42}$, S. Seidel ${ }^{35}$, Y. Seiya ${ }^{38}$, A. Semenov ${ }^{13}$, F. Sforza ${ }^{42, \text { hh }, ~ S . Z . ~ S h a l h o u t ~}{ }^{7}$, T. Shears ${ }^{27}$, P.F. Shepard ${ }^{43}$, M. Shimojima ${ }^{51, v}$, M. Shochet ${ }^{11}$, I. Shreyber-Tecker ${ }^{34}$, A. Simonenko ${ }^{13}$, P. Sinervo ${ }^{31}$, K. Sliwa ${ }^{52}$, J.R. Smith ${ }^{7}$, F.D. Snider ${ }^{15}$, A. Soha ${ }^{15}$, V. Sorin ${ }^{4}$, H. Song ${ }^{43}$, P. Squillacioti ${ }^{42, h h}$, M. Stancari ${ }^{15}$, R. St. Denis ${ }^{19}$, B. Stelzer ${ }^{31}$, O. Stelzer-Chilton ${ }^{31}$, D. Stentz ${ }^{15, x}$, J. Strologas ${ }^{35}$, G.L. Strycker ${ }^{32}$, Y. Sudo ${ }^{51}$, A. Sukhanov ${ }^{15}$, I. Suslov ${ }^{13}$, K. Takemasa ${ }^{51}$, Y. Takeuchi ${ }^{51}$, J. Tang ${ }^{11}$, M. Tecchio ${ }^{32}$, P.K. Teng ${ }^{1}$, J. Thom ${ }^{15, g}$, J. Thome ${ }^{10}$, G.A. Thompson ${ }^{22}$, E. Thomson ${ }^{41}$, D. Toback ${ }^{49}$, S. Tokar ${ }^{12}$,

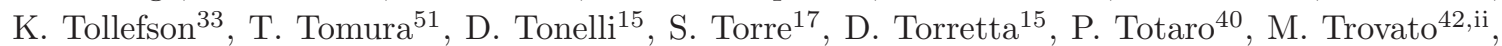

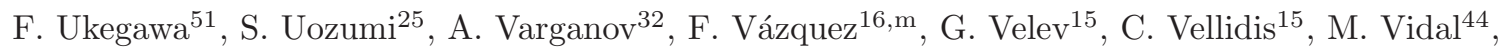
I. Vila ${ }^{9}$, R. Vilar ${ }^{9}$, J. Vizán ${ }^{9}$, M. Vogel ${ }^{35}$, G. Volpi ${ }^{17}$, P. Wagner ${ }^{41}$, R.L. Wagner ${ }^{15}$, T. Wakisaka ${ }^{38}$, R. Wallny ${ }^{8}$, S.M. Wang ${ }^{1}$, A. Warburton ${ }^{31}$, D. Waters ${ }^{28}$, W.C. Wester III ${ }^{15}$, D. Whiteson ${ }^{41, b}$, A.B. Wicklund ${ }^{2}$, E. Wicklund ${ }^{15}$, S. Wilbur ${ }^{11}$, F. Wick ${ }^{24}$, H.H. Williams ${ }^{41}$, J.S. Wilson ${ }^{36}$,

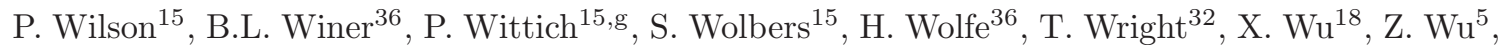
K. Yamamoto ${ }^{38}$, D. Yamato ${ }^{38}$, T. Yang ${ }^{15}$, U.K. Yang ${ }^{11, r}$, Y.C. Yang ${ }^{25}$, W.-M. Yao ${ }^{26}$, G.P. Yeh ${ }^{15}$, K. Yi ${ }^{15, n}$, J. Yoh ${ }^{15}$, K. Yorita ${ }^{54}$, T. Yoshida ${ }^{38,1}$, G.B. Yu ${ }^{14}$, I. Yu ${ }^{25}$, S.S. Yu ${ }^{15}$, J.C. Yun ${ }^{15}$, A. Zanetti ${ }^{50}$, Y. Zeng ${ }^{14}$, C. Zhou ${ }^{14}$, S. Zucchelli 6 ,ee

1 Institute of Physics, Academia Sinica, Taipei, Taiwan 11529, Republic of China

2 Argonne National Laboratory, Argonne, Illinois 60439, U.S.A.

3 University of Athens, 15771 Athens, Greece

4 Institut de Fisica d'Altes Energies, ICREA, Universitat Autonoma de Barcelona, E-08193, Bellaterra (Barcelona), Spain

5 Baylor University, Waco, Texas 76798, U.S.A.

6 Istituto Nazionale di Fisica Nucleare Bologna, Italy

7 University of California, Davis, Davis, California 95616, U.S.A.

8 University of California, Los Angeles, Los Angeles, California 90024, U.S.A.

9 Instituto de Fisica de Cantabria, CSIC-University of Cantabria, 39005 Santander, Spain

10 Carnegie Mellon University, Pittsburgh, Pennsylvania 15213, U.S.A.

11 Enrico Fermi Institute, University of Chicago, Chicago, Illinois 60637, U.S.A.

12 Comenius University, 84248 Bratislava, Slovakia; Institute of Experimental Physics, 04001 Kosice, Slovakia

13 Joint Institute for Nuclear Research, RU-141980 Dubna, Russia

14 Duke University, Durham, North Carolina 27708, U.S.A.

15 Fermi National Accelerator Laboratory, Batavia, Illinois 60510, U.S.A.

16 University of Florida, Gainesville, Florida 32611, U.S.A.

17 Laboratori Nazionali di Frascati, Istituto Nazionale di Fisica Nucleare, I-00044 Frascati, Italy

18 University of Geneva, CH-1211 Geneva 4, Switzerland

19 Glasgow University, Glasgow G12 8QQ, United Kingdom 
20 Harvard University, Cambridge, Massachusetts 02138, U.S.A.

21 Division of High Energy Physics, Department of Physics, University of Helsinki and Helsinki Institute of Physics, FIN-00014, Helsinki, Finland

22 University of Illinois, Urbana, Illinois 61801, U.S.A.

23 The Johns Hopkins University, Baltimore, Maryland 21218, U.S.A.

24 Institut für Experimentelle Kernphysik, Karlsruhe Institute of Technology, D-76131 Karlsruhe, Germany

25 Center for High Energy Physics: Kyungpook National University, Daegu 702-701, Korea; Seoul National University, Seoul 151-742, Korea; Sungkyunkwan University, Suwon 440-746, Korea; Korea Institute of Science and Technology Information, Daejeon 305-806, Korea; Chonnam National University, Gwangju 500-757, Korea; Chonbuk National University, Jeonju 561-756, Korea

26 Ernest Orlando Lawrence Berkeley National Laboratory, Berkeley, California 94720, U.S.A.

27 University of Liverpool, Liverpool L69 7ZE, United Kingdom

28 University College London, London WC1E 6BT, United Kingdom

29 Centro de Investigaciones Energeticas Medioambientales y Tecnologicas, E-28040 Madrid, Spain

30 Massachusetts Institute of Technology, Cambridge, Massachusetts 02139, U.S.A.

31 Institute of Particle Physics: McGill University, Montréal, Québec, Canada H3A 2T8; Simon Fraser University, Burnaby, British Columbia, Canada V5A 1S6; University of Toronto, Toronto, Ontario, Canada M5S 1A7; and TRIUMF, Vancouver, British Columbia, Canada V6T 2A3

32 University of Michigan, Ann Arbor, Michigan 48109, U.S.A.

33 Michigan State University, East Lansing, Michigan 48824, U.S.A.

34 Institution for Theoretical and Experimental Physics, ITEP, Moscow 117259, Russia

35 University of New Mexico, Albuquerque, New Mexico 87131, U.S.A.

36 The Ohio State University, Columbus, Ohio 43210, U.S.A.

37 Okayama University, Okayama 700-8530, Japan

38 Osaka City University, Osaka 588, Japan

39 University of Oxford, Oxford OX1 3RH, United Kingdom

40 Istituto Nazionale di Fisica Nucleare, Sezione di Padova-Trento, Italy

41 University of Pennsylvania, Philadelphia, Pennsylvania 19104, U.S.A.

42 Istituto Nazionale di Fisica Nucleare Pisa, Italy

43 University of Pittsburgh, Pittsburgh, Pennsylvania 15260, U.S.A.

44 Purdue University, West Lafayette, Indiana 47907, U.S.A.

45 University of Rochester, Rochester, New York 14627, U.S.A.

46 The Rockefeller University, New York, New York 10065, U.S.A.

47 Istituto Nazionale di Fisica Nucleare, Sezione di Roma 1, Italy

48 Rutgers University, Piscataway, New Jersey 08855, U.S.A.

49 Texas A $6 M$ University, College Station, Texas 77843, U.S.A.

50 Istituto Nazionale di Fisica Nucleare Trieste/Udine, I-34100 Trieste, Italy

51 University of Tsukuba, Tsukuba, Ibaraki 305, Japan

52 Tufts University, Medford, Massachusetts 02155, U.S.A.

53 University of Virginia, Charlottesville, Virginia 22906, U.S.A.

54 Waseda University, Tokyo 169, Japan

55 Wayne State University, Detroit, Michigan 48201, U.S.A.

56 University of Wisconsin, Madison, Wisconsin 53706, U.S.A.

57 Yale University, New Haven, Connecticut 06520, U.S.A.

With visitors from:

a Istituto Nazionale di Fisica Nucleare, Sezione di Cagliari, 09042 Monserrato (Cagliari), Italy

b University of CA Irvine, Irvine, CA 92697, U.S.A.

c University of CA Santa Barbara, Santa Barbara, CA 93106, U.S.A.

d University of CA Santa Cruz, Santa Cruz, CA 95064, U.S.A.

e Institute of Physics, Academy of Sciences of the Czech Republic, Czech Republic

f CERN, CH-1211 Geneva, Switzerland 
g Cornell University, Ithaca, NY 14853, U.S.A.

h University of Cyprus, Nicosia CY-1678, Cyprus

i Office of Science, U.S. Department of Energy, Washington, DC 20585, U.S.A.

j University College Dublin, Dublin 4, Ireland

k ETH, 8092 Zurich, Switzerland

1 University of Fukui, Fukui City, Fukui Prefecture, Japan 910-0017

$\mathrm{m}$ Universidad Iberoamericana, Mexico D.F., Mexico

$\mathrm{n}$ University of Iowa, Iowa City, IA 52242, U.S.A.

- Kinki University, Higashi-Osaka City, Japan 577-8502

p Kansas State University, Manhattan, KS 66506, U.S.A.

q Ewha Womans University, Seoul, 120-750, Korea

$r$ University of Manchester, Manchester M13 9PL, United Kingdom

s Queen Mary, University of London, London, E1 4NS, United Kingdom

t University of Melbourne, Victoria 3010, Australia

u Muons, Inc., Batavia, IL 60510, U.S.A.

v Nagasaki Institute of Applied Science, Nagasaki, Japan

w National Research Nuclear University, Moscow, Russia

x Northwestern University, Evanston, IL 60208, U.S.A.

y University of Notre Dame, Notre Dame, IN 46556, U.S.A.

z Universidad de Oviedo, E-33007 Oviedo, Spain

aa CNRS-IN2P3, Paris, F-75205 France

bb Texas Tech University, Lubbock, TX 79609, U.S.A.

cc Universidad Tecnica Federico Santa Maria, $110 v$ Valparaiso, Chile

dd Yarmouk University, Irbid 211-63, Jordan

ee University of Bologna, I-40127 Bologna, Italy

ff University of Padova, I-35131 Padova, Italy

gg University of Pisa

hh University of Siena

ii Scuola Normale Superiore, I-56127 Pisa, Italy

jj California Institute of Technology, Pasadena, California 91125, U.S.A.

kk INFN Pavia and University of Pavia, Italy 